\title{
Endogenia acadêmica: insights sobre a pesquisa brasileira
}

\author{
Tatiane Pelegrini ${ }^{1}$ \\ Marco Túlio Aniceto França ${ }^{2}$
}

\section{Resumo}

Este artigo analisa a endogenia acadêmica nas universidades públicas brasileiras, definida como a prática de recrutamento em que as universidades contratam os seus próprios doutorandos após a conclusão de seus estudos. Nesse sentido, foram utilizados dados dos docentes que atuam em grupos de pesquisa e o número de publicações em periódicos internacionais e nacionais, bem como o número de alunos de doutorado orientados como proxies da produtividade científica e da sobreposição das atividades de ensino e pesquisa. Por meio da aplicação de um modelo binomial negativo logit hurdle, os indivíduos que não são endógenos publicam ao menos um artigo internacional, mais artigos nacionais e possuem menos orientandos de doutorado. Contudo, não é possível afirmar categoricamente que os não endógenos são mais produtivos, uma vez que as diferentes áreas científicas possuem culturas disciplinares e de difusão distintas e o mercado de trabalho acadêmico brasileiro passa por uma fase de transição.

\section{Palavras-Chave}

Endogenia acadêmica. Pós-Graduação. Mobilidade. Logit hurdle.

\begin{abstract}
This article analyzes the academic inbreeding in Brazilian public universities, defined as a recruitment practice in which universities hire their own doctoral students upon completion of their studies. In this sense, we used data from professors working in research groups and the number of publications in international and national journals, as well as the number of

- Artigo extraído da tese de doutorado "O ensino superior no Brasil: contexto atual e mobilidade", apresentada à Pontifícia Universidade Católica do Rio Grande do Sul, Porto Alegre, RS, Brasil. Agradecemos à Adriane Santos, Luana Fraga e Matheus Serpa pelo auxílio com a provisão de dados. O presente trabalho contou com o apoio da Coordenação de Aperfeiçoamento de Pessoal de Nível Superior (CAPES), Código de Financiamento 001 e Conselho Nacional de Desenvolvimento Científico e Tecnológico (CNPq), Código de Financimento 312144-2019-9.

1 Pós Doutoranda - Pontifícia Universidade Católica do Rio Grande do Sul - Escola de Negócios End.: Avenida Ipiranga, 6681, Prédio 50 - Partenon - CEP: 90619-900 - Porto Alegre/RS - Brasil. E-mail: tatianepelegrini@pucrs.br - ORCID: https://orcid.org/0000-0002-8280-2247.

2 Professor - Pontifícia Universidade Católica do Rio Grande do Sul - Escola de Negócios. End.: Avenida Ipiranga, 6681, Prédio 50 - Partenon - CEP: 90619-900 - Porto Alegre/RS - Brasil. E-mail: marco.franca@pucrs.br - ORCID: http://orcid.org/0000-0003-1865-323X.

Recebido: 20/12/2019. Aceite: 20/08/2020

Editor responsável: Dante Mendes Aldrighi
\end{abstract}

(c) (i) (\$) Esta obra está licenciada com uma Licença Creative Commons Atribuição-Não Comercial 4.0 Internacional. 
doctoral students oriented as proxies for scientific research and the activities of teaching and research. By applying a negative binomial hurdle model, no-inbreds publish at least one international article, more national articles, and have less doctoral students under their supervision. However, it is not possible to state categorically that no-inbreds are more productive, since different scientific areas have different disciplinary cultures, and the academic market jobs is going through a transitional phase.

\section{Keywords}

Academic inbreeding. Postgraduate. Mobility. Logit hurdle.

\section{JEL Classification}

C24. 123. 128.

\section{Introdução}

As recentes políticas públicas voltadas ao ensino superior promoveram também uma expansão da pós-graduação brasileira e implicaram a ampliação do número de programas de pós-graduação e maior distribuição de bolsas de auxílio, além da titulação de um alto índice de mestres e doutores. ${ }^{1}$ Paralelamente ao novo Plano de Educação foi elaborado o Plano Nacional de Pós-Graduação 2011-2020, com o objetivo de definir novas diretrizes, estratégias e metas para dar continuidade e avançar nas propostas de política de pós-graduação e pesquisa no Brasil.

Desde a sua implementação pelo governo militar, o maior desafio do sistema de ensino da pós-graduação no Brasil é a formação de recursos humanos a fim de capacitar os docentes das universidades, integrar a pós-graduação no sistema universitário, valorizar as ciências básicas e evitar disparidades regionais. De acordo com a agência de Coordenação e Aperfeiçoamento de Pessoal de Nível Superior (Capes 2019), atualmente, o país possui 288.593 estudantes de pós-graduação, dos quais 131.607 são de mestrado acadêmico, 42.064 são de mestrado profissional e 114.867 são de doutorado, distribuídos em 4.291 programas.

1 De acordo com o Sistema de Informações Georreferenciadas, desenvolvido pela Coordenação e Aperfeiçoamento de Pessoal de Nível Superior (Geocapes 2019), entre 2000 e 2018, o número de programas de pós-graduação apresentou um crescimento de, aproximadamente, $66,5 \%$; a concessão de bolsas, para o mesmo período, cresceu $79,7 \%$, bem como o número de titulados $(73,5 \%)$. 
Nos últimos anos, cada vez mais alunos têm feito a transição direta graduação/pós-graduação, tanto em função do aumento da oferta de cursos, mas sobretudo pelo aumento da demanda da sociedade por um maior nível de escolarização (Barbosa et al. 2018; Cirani et al. 2015). A criação de novos cursos também estimulou a busca pela excelência e novos conhecimentos voltados à inovação e interdisciplinaridade, evitando a endogenia na formação dos docentes (Brasil 2010). O trabalho seminal de Berelson (1960) define essa endogenia como inbreeding acadêmico, que consiste no recrutamento de acadêmicos pela mesma instituição na qual foi realizado o doutorado.

O conceito de endogenia está relacionado à imobilidade no corpo docente e pode afetar a produtividade científica, bem como a excelência e inovação ao limitar a troca de ideias e a circulação de conhecimento gerada pelas redes de colaboração entre países e instituições (Horta et al. 2010). As atividades científicas são distribuídas de modo desigual no espaço geográfico (Royal Society 2011) e, nesse sentido, o Brasil apresenta uma heterogeneidade significativa com a concentração das atividades relacionadas à localização dos campi das universidades públicas - padrão apresentado por países em desenvolvimento (Sidone et al. 2017).

De acordo com Boschma (2005), além da proximidade geográfica, outras dimensões de proximidade, como institucional e organizacional, reforçam os impactos da primeira e contribuem para o incremento da produção científica/inovações, bem como de sua qualidade. A endogenia acadêmica, nesse contexto, pode estar vinculada às noções de proximidade, sendo que uma avaliação quantitativa do número de publicações pode contribuir, em um primeiro momento, para que a atividade científica seja examinada no Brasil.

O Brasil, como nação cientificamente emergente, promove o crescimento de sua produção científica especialmente por meio de uma maior colaboração interna (Royal Society 2011). A endogenia, nesse contexto, pode ser considerada pequena e periférica da profissão acadêmica, embora seja bastante difundida globalmente e apresente, inclusive, aspectos positivos, como maior colaboração entre pares, continuidade dos valores e práticas institucionais, bem como a manutenção dos próprios talentos na instituição (Altbach et al. 2015). 
Dessa forma, este ensaio dedica-se a analisar outra faceta da mobilidade estudantil, relativa à transição entre pós-graduação e carreira docente nas universidades brasileiras, a fim de determinar se a endogenia acadêmica afeta a produtividade científica. Essa análise, de acordo com o nosso conhecimento, é inédita para o Brasil e relevante em função de seu estágio de desenvolvimento, concentração de universidades e ausência de incentivos econômicos à mobilidade de docentes para instituições públicas, uma vez que os salários são essencialmente os mesmos.

Também se almeja contribuir para a literatura cientométrica brasileira por meio da análise das publicações científicas e grau de endogeneidade que os pesquisadores das instituições públicas do país apresentam. A avaliação do desempenho científico e paradigmas individuais de publicação é tarefa desafiadora (Haddad et al. 2017) e possibilita com que se estabeleçam padrões em relação à produção da ciência e seus sistemas de comunicação (Mingers e Leydesdorff 2015).

A fim de cumprir este propósito, este trabalho está dividido em mais três seções. A Seção 2 apresenta e discute a literatura acerca dos aspectos da endogenia acadêmica no ensino superior, bem como a literatura cientométrica. A Seção 3 apresenta os dados a utilizar na análise e o modelo binomial negativo logit hurdle, metodologia empregada para a obtenção dos resultados que, posteriormente, são analisados e discutidos na Seção 4. Por fim, a quinta seção explicita as considerações finais da pesquisa.

\section{Revisão de literatura}

\subsection{Endogenia acadêmica}

A endogenia acadêmica vem sendo abordada desde a década de 1930, como um elemento na caracterização das universidades dos Estados Unidos (McNeely 1932; Reeves et al. 1933; Eelss e Cleveland 1935; Hollingshead 1938, Wilson 1942). Esses estudos demonstraram, sem estimações estatísticas, que faculdades com endogenia apresentavam menores níveis de produção quando comparadas a faculdades sem endogenia. Na década de 1950, houve a tentativa de mensurar empiricamente a endogenia acadêmi$\mathrm{ca}$, o que levou à necessidade de definir mais precisamente o seu conceito. 
Desse modo, Berelson (1960) forneceu uma definição mais estrita de endogenia acadêmica, que consiste no recrutamento de acadêmicos pela mesma instituição na qual foi realizado o doutorado, conceito relacionado à imobilidade. Nesse período, McGee (1960) realizou a primeira análise empírica entre endogenia acadêmica e a produtividade científica para a Universidade do Texas, inferindo que essa relação é positiva e tem utilidade na instituição de ensino.

A fim de testar a hipótese de Berelson e McGee, de que a endogenia institucional apresenta um padrão generalizado de recrutamento, Hargens e Far (1973) constataram relações pequenas e consistentemente negativas entre a endogenia e a produtividade acadêmica. Para Wyer e Conrad (1984), essa relação não foi significativamente negativa, embora quando aplicados controles sobre o tempo na academia, docentes endógenos obtiveram maior produtividade, fato relacionado a características individuais superiores.

A ocorrência de endogenia pode também ser considerada uma forma extrema de imobilidade acadêmica (Berelson 1960; Dutton 1980; Horta 2013). Muitos países não possuem um mercado de trabalho para empregos acadêmicos, além de pouca ou nenhuma tradição em mobilidade (Altbach et al. 2015; Stephan e Ma 2005).

O Brasil pode figurar entre esses países, uma vez que os salários dos professores das universidades federais são essencialmente os mesmos, e estes estão localizados majoritariamente em grandes centros econômicos, ou seja, não há incentivo à mobilidade, que é intrinsecamente relacionada a melhor qualidade e produtividade em pesquisa (Dillon 2003). Um cenário em que os salários são rígidos não propicia incentivos econômicos para estimular a mobilidade, fazendo com que o escopo de seleção seja mais local (Horta e Yudkevich 2016).

De acordo com Tavares et al. (2017), a produtividade é o tema principal do tópico relativo à endogenia, em que aspectos das universidades americanas em relação à mobilidade e trajetória da carreira acadêmica tornou-se relevante em outros países, apreensivos por um nepotismo velado. De modo geral, a endogenia tem sido reportada na literatura como prejudicial por uma série de razões (Tavares et al. 2017; Tavares et al. 2015; Horta 2013; Horta et al. 2010; Godechot e Louvet 2010; Cruz-Castro e SanzMenéndez 2010). 
Dentre os efeitos negativos em recrutar os próprios alumni, destaca-se a influência sobre a produtividade acadêmica, que é reduzida para professores que são endógenos, seja no número de artigos científicos (Horta et al. 2010), citações (Eisenberg e Well 2000) ou artigos em revistas nacionais de maior classificação (Sivak e Yudkevich 2015). Demais evidências têm demonstrado que esses profissionais também apresentam maior carga horária de ensino e menos bolsas de pesquisa (McGee 1960), além de atuarem em programas com menor excelência (Hargens e Farr 1973; Eells e Cleveland 1935; Reeves et al. 1933).

Além da quantidade de trabalhos produzidos, normalmente a qualidade e a capacidade inovadora são abordadas na literatura. Parte-se do pressuposto que instituições com maiores coeficientes de endogenia tendem a ser mais "locais", ou seja, sua orientação é menos vinculada ao mundo acadêmico e à pesquisa e mais a atividades de ensino e administrativas (Wyer e Conrad 1984; Hollingshead 1938). As publicações, desse modo, tendem a ser orientadas para revistas locais e de menor qualidade (Altbach et al. 2015; Gorelova e Yudkevich 2015; Horta 2013; Velho e Krige 1984).

No entanto, os endógenos não necessariamente são menos produtivos quando comparados aos não endógenos (Yudkevich et al. 2015; Clark e Larson 1972; Dutton 1980). Os aspectos positivos que são reportados na literatura, especialmente nos estágios iniciais, são a estabilidade e a colaboração entre pares (Gorelova e Lovakov 2016; Horta et al. 2011). Outro ponto é a perpetuação dos valores e práticas institucionais com vistas a preservar a identidade das universidades (Lafferty 1964) e manter os seus talentos (Pan 1993).

As universidades atuam no sentido de reproduzir as práticas e o conhecimento adquirido, além de consolidar estruturas sociais (Yamanoi 2005). Essa prática é tida como extremamente comum e, inclusive, é considerada uma questão de orgulho das instituições que podem reter os seus talentos intelectuais (Altbach et al. 2015). No entanto, é possível denotar uma mudança na forma de contratação, que prioriza as conexões sociais aos méritos acadêmicos (Rocca 2007), criando um ambiente nepótico (Godechot e Louvet 2010).

A contratação de docentes apresenta formas variadas de acordo com a natureza das universidades, nesse sentido as universidades públicas brasileiras devem abrir editais públicos de seleção com critérios de avaliação preestabelecidos pelos departamentos, procedimentos que de- 
vem restringir a endogenia. Entretanto, Altbach et al. (2015) apontam que a endogenia não pode ser eliminada com a introdução de requisitos formais abertos e não discriminatórios para candidatos externos. Ainda de acordo com os autores, há uma grande lacuna entre os procedimentos formais e os realmente praticados:

"While formal procedures may exist, and even be supported by such mechanisms as the publication of open calls in national newspapers, real practices are guided by entrenched informal conventions and routines that limit the hiring of outsiders. So, when asked why inbreeding is a prevailing reality in their institutions, university administrators often refer to the fact that no outside applications are received, which explains the pervasiveness of internal hiring." (Altbach et al. 2015, p. 324).

Em relação ao Brasil, o primeiro trabalho sobre endogenia acadêmica é de Velho e Krige (1984), em uma análise qualitativa de cientistas agrícolas. Foi constatado que os cientistas de maior renome se comunicam em menor grau com cientistas de outras instituições e tendem a interagir com colegas da própria organização. De modo geral, os trabalhos voltados para o contexto brasileiro enfocam cursos e instituições específicas com análises descritivas de pequenas amostras, como Braga e Venturini (2013), que buscaram identificar a endogenia acadêmica em um programa de pós-graduação em Direito de uma universidade pública do Estado de São Paulo.

Mais recentemente, Barbosa et al. (2018) verificaram a relação entre a endogenia na formação dos coordenadores e os conceitos Capes dos Programas de pós-graduação em ciências contábeis no Brasil com o método de análise de correspondências. Os resultados evidenciaram que há associação positiva entre endogenia e os conceitos atribuídos pela Capes, embora universidades com cursos mais tradicionais apresentem menores níveis de produção científica em razão da endogenia de seus docentes.

É importante salientar a distinção entre as universidades públicas e privadas, em que as primeiras devem cumprir normativas legais preestabelecidas e universidades privadas possuem os próprios critérios de contratação. Atualmente, as universidades privadas correspondem a $18,5 \%$ dos progra- 
mas de pós-graduação no Brasil e possuem disposições legais diferenciadas ao estarem sob a influência de demandas mercadológicas e diretrizes institucionais.

De modo geral, as instituições privadas estão voltadas para uma estrita formação profissional e empregam docentes com pequenos volumes de titulação acadêmica dedicados, na maior parte do tempo, às atividades de ensino. Em seu interior há uma ausência de estruturas orientadas para pesquisa e pós-graduação strictu sensu e a qualidade dos cursos ofertados é menor quando comparada às universidades públicas (Yudkevich 2014; Martins 2009).

Outra distinção deve ser feita em relação à esfera das universidades federais e estaduais brasileiras. Em comum, as instituições possuem a oferta gratuita de cursos de graduação e pós-graduação, entretanto, o que as diferencia é a origem da verba para manutenção das atividades. Os salários dos professores das universidades federais são padronizados, enquanto os salários das estaduais têm modificações entre as unidades da federação.

\subsection{Cientometria}

$\mathrm{Na}$ literatura da cientometria, definida como o desenvolvimento de “(...) métodos quantitativos da pesquisa sobre o desenvolvimento da ciência como processo informacional" (Nalimov e Mul'chenko 1971, p. 2), a concepção de uma ideia e sua sistematização em formato de publicação é tida como uma tarefa complexa (Sahu e Panda 2014). De modo geral, a cientometria analisa a ciência como um sistema de comunicação diferençável pelos materiais e métodos empregados (Mingers e Leydesdorff 2015; Sahu e Panda 2014).

A cientometria aborda aspectos relativos ao desenvolvimento da ciência e da tecnologia, entretanto, a noção central dessa área teve como ponto de partida as citações (Mingers e Leydesdorff 2015). Frequentemente, são utilizadas como meio de avaliar a qualidade da produção científica, em reconhecimento pelos colegas e valorização pela contribuição na área de investigação, as citações podem ser consideradas indicadores de atraso ou, por vezes, brutas (Royal Society 2011). 
Nesse sentido, mensurar o valor e os impactos da ciência é um desafio para a comunidade científica, em que existem controvérsias em relação à influência das métricas utilizadas em sua valoração (Haddad et al. 2017; Royal Society 2011). A medida de qualidade das publicações, denominada bibliometria, pode ser expressa por vários indicadores (Durieux e Gevenois 2010), e revestir-se de importância para pesquisadores e organizações na previsão de sucesso (Wang et al. 2019) e financiamento da pesquisa científica (Ravenscroft et al. 2017).

De acordo com Durieux e Gevenois (2010), os indicadores bibliométricos podem ser classificados em três categorias: quantidade (mensuram a produtividade); desempenho (mensuram a qualidade); e estruturais (mensuram as conexões entre publicações, autores e campos científicos). Wu (2019, p. 5) sistematiza a cientometria em uma rede fundamental de três camadas, sendo que os pesquisadores, questões de pesquisa e suas atividades científicas são os principais atores dessa rede.

Logo, a primeira camada é composta por autores e inventores; a segunda por artigos e patentes; e a terceira pelas tecnologias e conceitos desenvolvidos. Entre as camadas existem conexões, como as relações sociais e acadêmicas (orientação e colaboração); entre artigos (citações) e entre conceitos (lógica construída a partir da interação entre as áreas do conhecimento). Essa configuração permite que a cientometria seja compreendida como uma rede global de interação e colaboração.

Dado que a proximidade geográfica desempenha um papel direto na colaboração (Sahu e Panda 2014) e aumento da produção científica (Frenken $e t$ al. 2009; Ponds et al. 2009; Boschma 2005), a abordagem espacial também é muito presente nessa área. A proximidade institucional (de universidades, indústria e órgãos governamentais) retrata a uniformidade das estruturas de incentivo que alinham os objetivos dos pesquisadores (Frenken $e t$ al. 2009). O aumento esperado de colaborações entre os autores, dada a sua proximidade, resulta em um incremento na qualidade de publicações (Sahu e Panda 2014).

Em relação ao Brasil, tem sido analisado o grau de colaboração entre pesquisadores (Haddad et al. 2017; Mena-Chalco et al. 2014) bem como o papel da geografia do país na produção e difusão de conhecimento (Sidone et al. 2017). Existe uma grande dificuldade em coletar dados relativos a 
publicações, o que impede o avanço desse campo de estudo e a aplicação de análises empíricas no Brasil (Mena-Chalco et al. 2014).

Ainda segundo Mena-Chalco et al. (2014), a grande maioria dos estudos voltados à análise e avaliação da produção científica brasileira são orientados para áreas do conhecimento específicas. Nesse sentido, citam-se trabalhos na área da computação (Bazzan e Argenta 2011; Laender et al. 2008); educação (Coutinho et al. 2012), economia (Haddad et al. 2017; Guimarães 2011; Faria et al. 2007) e ciências biológicas (Zorzetto et al. 2006). Mais recentemente, a produção das universidades em termos de pesquisa e inovação tem sido contemplada pela cientometria brasileira (Faria et al. 2018).

A análise da endogenia acadêmica por meio da avaliação quantitativa da produção científica no contexto atual de pós-graduação constitui-se como a contribuição deste trabalho no campo da literatura cientométrica brasileira. Conforme supracitado, a endogenia no corpo docente relacionase com a produtividade e a qualidade da pesquisa, portanto, a seção a seguir designa os procedimentos metodológicos utilizados para determinar se ocorre endogenia acadêmica e, em que grau esse fenômeno afeta a produtividade de pesquisa científica.

\section{Metodologia}

\subsection{Modelos de contagem}

Os modelos de contagem acomodam os casos em que a variável dependente é um número inteiro não negativo, sendo um evento a realização desta variável estocástica. Essas particularidades requerem o uso de modelos especiais de regressão, uma vez que modelos tradicionais lineares são deficientes em prever a natureza desses dados (mesmo com a transformação das variáveis), exceto para os casos em que se aproximem de uma distribuição normal por meio de uma média elevada (Vives et al. 2006; Cameron e Trivedi 1998). 
Entre as alternativas mais plausíveis estão o uso de binomiais negativos de zeros inflados (ZINB) ou binomiais negativos logit hurdle (NBLH) em decorrência do excesso de limitações apresentadas pelos modelos de Poisson, mais recorrentes nos manuais de estatística (Loyes et al. 2012; Hilbe 2011). Em termos das variáveis utilizadas (publicações e orientações), a metodologia considerada mais adequada pela literatura é o modelo NBLH, uma vez que permite a divisão da variável dependente em dois grupos com a probabilidade de apresentar uma publicação/orientação ou maior número de publicações/orientações (Tavares et al. 2019; Alipova e Lovakov 2018; Loeys et al. 2012).

Dessa forma, o objetivo deste trabalho é analisar a relação entre a produtividade científica e a endogenia acadêmica. A fim de embasar o modelo empírico, é realizada uma regressão binomial negativa logit hurdle:

$$
Y_{i j k}=x_{i j k}^{\prime} \beta+\alpha_{j}+\gamma_{j}+\delta_{k}+e_{i j k}
$$

em que $Y_{i j k}$ é a produção/atividade do acadêmico $i$ para a universidade $j$ para a área científica $k$, a saber: total de artigos publicados em periódicos internacionais e nacionais e total de orientações de doutorado. A variável independente $\left(x_{i j k}^{\prime}\right)$ inclui variáveis dummies para as categorias de pesquisadores endógenos e não endógenos. As outras variáveis dependentes são de controle, incluindo efeitos fixos para o docente $\left(\gamma_{j}\right)$; Programa de Pós-Graduação $\left(\alpha_{j}\right)$ e área científica $\left(\delta_{k}\right)$.

\subsection{O modelo}

Nos modelos binomiais negativos logit hurdle, os valores de contagem zero e não zero são claramente separados. A parte de contagem zero analisa o efeito de pertencer a grupos com pelo menos uma publicação/aluno orientado e a parte de contagem (não zero) o efeito de pertencer ao grupo de endógenos no total de publicações e alunos orientados de doutorado. Assim sendo, o modelo hurdle é uma mistura finita, composta pela combinação de zeros, gerados por uma densidade com zeros, e números positivos, gerados por outra densidade truncada. 
Modelos hurdle ou de duas partes, relaxam a suposição de que os zeros e os dados de contagem sejam provenientes do mesmo processo de geração de dados (Cameron e Trivedi 2010). Os zeros são determinados pela função de densidade $f_{1}(\cdot)$, de modo que $\operatorname{Pr}(y=0)=f_{1}(0)$ e $\operatorname{Pr}(Y>0)=1-f_{1}(0)$.

A contagem positiva deriva da densidade truncada $f_{2}(y \mid y>0)=f_{2}(y) /$ $\left\{1-f_{2}(0)\right\}$ multiplicado por $\operatorname{Pr}(Y>0)$ para garantir que a soma das probabilidades seja igual a um. De modo notacional:

$$
f(y)=\left\{\begin{array}{lr}
f_{1}(0) & \text { se } y<0 \\
\frac{1-f_{1}(0)}{1-f_{2}(0)} f_{2}(y) & \text { se } y \geq 1
\end{array}\right.
$$

Cameron e Trivedi (2010) destacam que, embora a motivação desse modelo é manipular zeros em excesso, também é capaz de modelar poucos zeros. A interpretação do modelo é de que os indivíduos tomam decisões em dois estágios, cada parte compondo um modelo de decisão. As duas partes do modelo são funcionalmente independentes, portanto, a estimação do modelo pode, inicialmente, maximizar os dois termos de probabilidades, o que corresponde aos zeros (toda a amostra) e outro a respeito dos dados positivos (observações de contagem).

Os modelos hurdle alteram as especificações da média condicional representada pela Equação (3):

$$
E(y \mid x)=\operatorname{Pr}\left(y_{1}>0 \mid x_{1}\right) * E_{y_{2>0}}\left(y_{2} \mid y_{2}>0, x_{2}\right)
$$

Relacionado especificamente ao modelo hurdle binomial negativo, a função de variância é quadrática (via distribuição binomial negativa - NB2) e apresenta $\mu_{i 1}=\exp \left(x_{i}^{\prime} \beta_{1}\right)$ para o parâmetro de distribuição de zeros e $\mu_{i 2}=\exp \left(x_{i}^{\prime} \beta_{2}\right)$ para o conjunto de números positivos, $J=\{1,2, \ldots\}$. Ao se definir uma função indicadora $\left(I\left[y_{i} \in J\right]=1\right.$ se $y_{i} \in J$ e $\left[y_{i} \in J\right]=0$ se $\left.y_{i}=0\right)$, obtém-se a distribuição NB2:

$$
\operatorname{Pr}\left[y_{i}=0 \mid x_{i}\right]=\left(1+\alpha_{i} \mu_{i 1}\right)^{-1 / \alpha_{1}}
$$

$\operatorname{Pr}\left[y_{i} / x_{i}, y_{i}>0\right]=\frac{\Gamma\left(y_{i}+\alpha_{2}^{-1}\right)}{\Gamma\left(\alpha_{2}^{-1}\right) \Gamma\left(y_{i}+1\right)}\left(\frac{1}{\left(1+\alpha_{2} \mu_{i 2}\right)^{1 / \alpha_{2}}-1}\right)^{-\alpha_{2}^{-1}}\left(\frac{\mu_{i 2}}{\mu_{i 2}+\alpha_{2}^{-1}}\right)^{y_{i}}$ 
em que $x$ se refere à probabilidade de se obter zeros e $y$ à probabilidade condicional da distribuição NB2 truncada; qualquer modelo binário pode ser usado para modelar o resultado zero versus positivo. Quando $\alpha_{1}=1$, a função de probabilidade apresenta-se como a equação abaixo:

$$
\operatorname{Pr}\left[y_{i}=0 \mid x_{i}\right]=\left(1+\mu_{i 1}\right)^{-1}
$$

de forma que: $\mu_{1 i}=\exp \left(x_{1 i}^{\prime} \beta_{1}\right)$, o primeiro estágio do modelo hurdle é um modelo logit de escolha binária, popularmente mais utilizado. Em suma, o modelo apresenta dois componentes, um de contagem truncada - binomial negativo - utilizado para os valores positivos e um componente binário hurdle - que modela os dados de contagem versus zeros. Assim sendo, no presente trabalho, o modelo NBLH foi utilizado a fim de comparar o número de publicações e alunos de doutorado orientados para os acadêmicos endógenos e não endógenos, sendo apresentados as partes de contagem (não zero) e zero separadamente.

O método indicado para comparar a qualidade de ajuste dos modelos que são não "aninhados", como o caso de modelos específicos de contagem, é a comparação dos critérios de informação de Akaike (AIC). Um menor valor de AIC indica melhor qualidade de ajustamento aos dados, sendo que a Tabela Al (em Anexo) demonstra que o melhor modelo para prever os números de artigos publicados em periódicos internacionais e nacionais e de doutorandos orientados é o NBLH em comparação com os modelos de Poisson de zeros inflados (ZIP) e logit hurdle (PLH), bem como o binomial negativo de zeros inflados (ZINB).

\subsubsection{Fonte de dados}

Os dados foram extraídos da plataforma Geocapes, do conjunto de dados abertos da Capes e dos arquivos dos censos do diretório de grupos de pesquisa disponibilizados pelo Conselho Nacional de Desenvolvimento Científico e Tecnológico (CNPq). Os censos reúnem informações sobre os recursos humanos constituintes dos grupos (pesquisadores, estudantes e técnicos), a produção científica, linhas de pesquisa em andamento e especialidades do conhecimento a fim de traçar um perfil geral da atividade científica brasileira. 
A Tabela 1 elenca as demais variáveis utilizadas no modelo, bem como o teste de diferença de médias para as variáveis utilizadas, que demonstra a semelhança entre os grupos na maioria das características. Ademais, salienta-se que uma série de especificações foram realizadas a fim de compor a base de dados; inicialmente, foram omitidos pesquisadores de programas de mestrado e demais cursos da modalidade profissional. Os cursos acadêmicos objetivam a formação de pesquisadores, consubstanciada na oferta do doutorado; e cursos profissionalizantes formam profissionais para o desempenho de funções distintas da pesquisa acadêmica (Brasil 2001).

Também foi observada a natureza pública das universidades, uma vez que todas as instituições públicas devem cumprir normativas legais para o recrutamento e a nomeação acadêmica, assim, pesquisadores de instituições privadas foram desconsiderados em função dos critérios de seleção subjetivos e diversos (Tavares et al. 2019). Outro ponto diz respeito à diversificação na oferta, em que foram consideradas apenas as instituições ${ }^{2}$ em que são oferecidos cursos das mesmas áreas disciplinares de avaliação da Capes (Tavares et al. 2015; Cruz-Castro e Sanz-Menéndez 2010).

Em seguida, foram selecionados pesquisadores com o título de doutorado atuando em programas com, no mínimo, 5 anos de existência e em funcionamento, uma vez que um menor período não constituiria tempo hábil ao PPG de formar e recrutar os próprios doutorandos (Horta 2013). Embora a base de dados informe o período de tempo entre a conclusão do doutorado e o tempo presente, não foi possível excluir acadêmicos que previamente trabalharam em outra universidade depois de terem concluído o doutorado, os chamados "cordões de prata" (Horta 2013; Berelson 1960).

Assim sendo, este trabalho considera como endógenos os docentes que atuam profissionalmente na mesma universidade em que foi realizado o doutoramento, sem contemplar a origem dos demais títulos acadêmicos (Tavares et al. 2019; Cruz-Castro e Sanz-Menéndez 2010; Horta et al. 2010; Einsenberg e Wells 2000; Wyer e Conrad 1984; Berelson 1960). Essa diferenciação é concebida na literatura, sendo que as categorias são definidas por um elemento analítico essencial: mobilidade entre instituições - não endógenos - ou sua falta durante a carreira - endógenos (Horta 2013). Ao assumir tal denominação, a amostra final é composta por 17.614 docentes, sendo 7.186 endógenos (40,8\%) e 10.428 não endógenos (59.20\%).

2 Tabela A2 (em Anexo). 
Em relação às variáveis restantes (Tabela 1), destaca-se que a ênfase avaliativa incide sobre os produtos das atividades dos pesquisadores, principalmente a produção bibliográfica qualificada (Horta e Moraes 2005). Assim sendo, as variáveis de produtividade científica utilizadas são o número de teses de doutorado concluídas que foram supervisionadas (combinam elementos de pesquisa e ensino) e o número de artigos publicados em periódicos internacionais e nacionais avaliados por pares entre 2014 e 2016.

De acordo com Hirsch (2005), a produtividade científica pode ser definida como o resultado de uma série de atividades de pesquisa, como a publicação de artigos nacionais e internacionais, livros e capítulos de livros, obtenção de bolsas de pesquisa, orientação de alunos, produção de patentes, participação como membro de corpo editorial, entre outros. A performance em pesquisa e produtividade tem sido mensurada por meio da quantidade de produtos de pesquisa, como a publicação de artigos em revistas avaliadas por pares (Horta 2013; Horta et al. 2010).

As publicações são computadas durante um intervalo de 3 anos, período de tempo cuja literatura que aborda produção e carreira científica considera mais adequado para avaliar o comportamento das publicações recentes (Horta 2013), além de controlar os efeitos do tempo de carreira docente ou "senioridade acadêmica" (Horta e Veloso 2010). De acordo com Fox (2005), esse período de tempo alberga as atividades de trabalho voltadas ao exercício de pesquisa, submissão e publicação. A divisão entre periódicos de abrangência internacional e nacional busca captar a qualidade ou inovação da pesquisa (Sivak e Yudkevich 2015; Birnbaum 2005).

A análise considera ainda uma série de variáveis de controle identificadas pela literatura que afetam a produtividade científica, como gênero, anos desde que o título de doutorado foi obtido, país em que o título de doutorado foi obtido (classificados como Brasil / Estrangeiro), se o docente possui bolsa de produtividade em pesquisa e a alocação das atividades sob a forma de professor permanente e professor colaborador.

As bolsas de produtividade em pesquisa são benefícios financeiros concedidos a pesquisadores que possuam produção científica, tecnológica e de inovação de destaque em suas respectivas áreas do conhecimento com o objetivo de reconhecer o trabalho e incentivar o aumento da produção dos pesquisadores. A bolsa de produtividade em pesquisa é organizada em níveis que provêm complementações salariais crescentes: $2,1 \mathrm{D}, 1 \mathrm{C}, 1 \mathrm{~B}$, 
1A, sendo que os últimos quatro níveis são coletivamente chamados de "níveis 1" (mais altos).

Os docentes permanentes desenvolvem atividades de ensino na pós-graduação e/ou graduação, participam de projetos de pesquisa, orientação de alunos de mestrado ou doutorado e possuem um vínculo funcional-administrativo com a instituição. A categoria de colaboradores inclui os demais membros do corpo docente que participam sistematicamente do desenvolvimento de projetos de pesquisa ou atividades de ensino ou extensão e/ou da orientação de estudantes, incluídos bolsistas de pós-doutorado, independentemente de possuírem ou não vínculo com a instituição (Capes 2016).

Características dos Programas de Pós-Graduação (PPGs) também foram incluídas, como sua data de criação e o conceito atribuído pela avaliação quadrienal da Capes, que se distribuem em notas 3 (regular), 4 (bom) e 5 (muito bom), e destes últimos se destacam programas excelentes, com notas 6 e 7, que constituem referências para as áreas. ${ }^{3}$ Em relação à localização dos PPGs foi incluída uma variável que diferencia aqueles situados em capitais estaduais. Esse controle é inserido a fim de analisar a heterogeneidade espacial das atividades científicas, presente em países em desenvolvimento e nas capitais desses países (Sidone et al. 2016; Royal Society 2011).

Por fim, as áreas de avaliação contempladas pela Capes ${ }^{4}$ seguem na Tabela 1, uma vez que estas controlam as culturas disciplinares e padrões de publicação específicos de cada área, evitando que os resultados sejam viesados em razão de heterogeneidades não observadas (Horta 2013).

3 Programas que recebem notas 1 e 2 deixam de ser credenciados pela Capes, ou seja, entram em desativação.

4 A área de avaliação Multidisciplinar foi excluída da análise por sua característica plural e relação colinear com as demais áreas. 


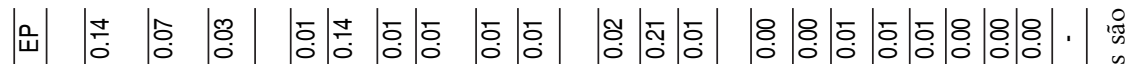

\begin{tabular}{|c|c|c|c|c|c|c|c|c|c|c|c|c|c|c|c|c|c|c|c|c|}
\hline 言 & | & @ִ & 음 & 㒸 & $\frac{\bar{\sigma}}{\bar{\top}}$ & דִ̣ & סִ & 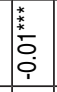 & 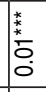 & ơ & & $\cong$ & 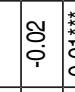 & & & \begin{tabular}{l}
8 \\
\hdashline \\
\end{tabular} & & & 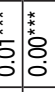 & \\
\hline 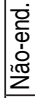 & $\mid \begin{array}{l}\infty \\
0 \\
i\end{array}$ & 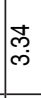 & 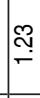 & fó & 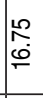 & ஸ़ & 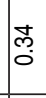 & $\left|\begin{array}{l}\mathscr{m} \\
0 \\
0\end{array}\right|$ & $\frac{1}{0}$ & $\underset{i \infty}{E}$ & 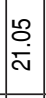 & שֶ. & $\stackrel{\cong}{\hdashline}$ & $\frac{}{\circ}$ & $\stackrel{\Upsilon}{\circ}$ & $\frac{1}{0}$ & ְ. & & : & 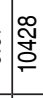 \\
\hline |் & 粂 & : & $\stackrel{m}{\rightleftarrows}$ & 电 & 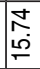 & 0 & ల్ల & $\mid \begin{array}{c}\tilde{\infty} \\
0 \\
0\end{array}$ & $\frac{\infty}{0}$ & $\mid \begin{array}{l}\bar{\infty} \\
i \infty \\
100\end{array}$ & 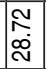 & 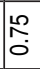 & : & $\bar{\sigma}$ & ָָ & $\frac{\sim}{\circ}$ & m. & & $\stackrel{0}{\circ}$ & $\stackrel{\infty}{\frac{\infty}{R}}$ \\
\hline
\end{tabular}

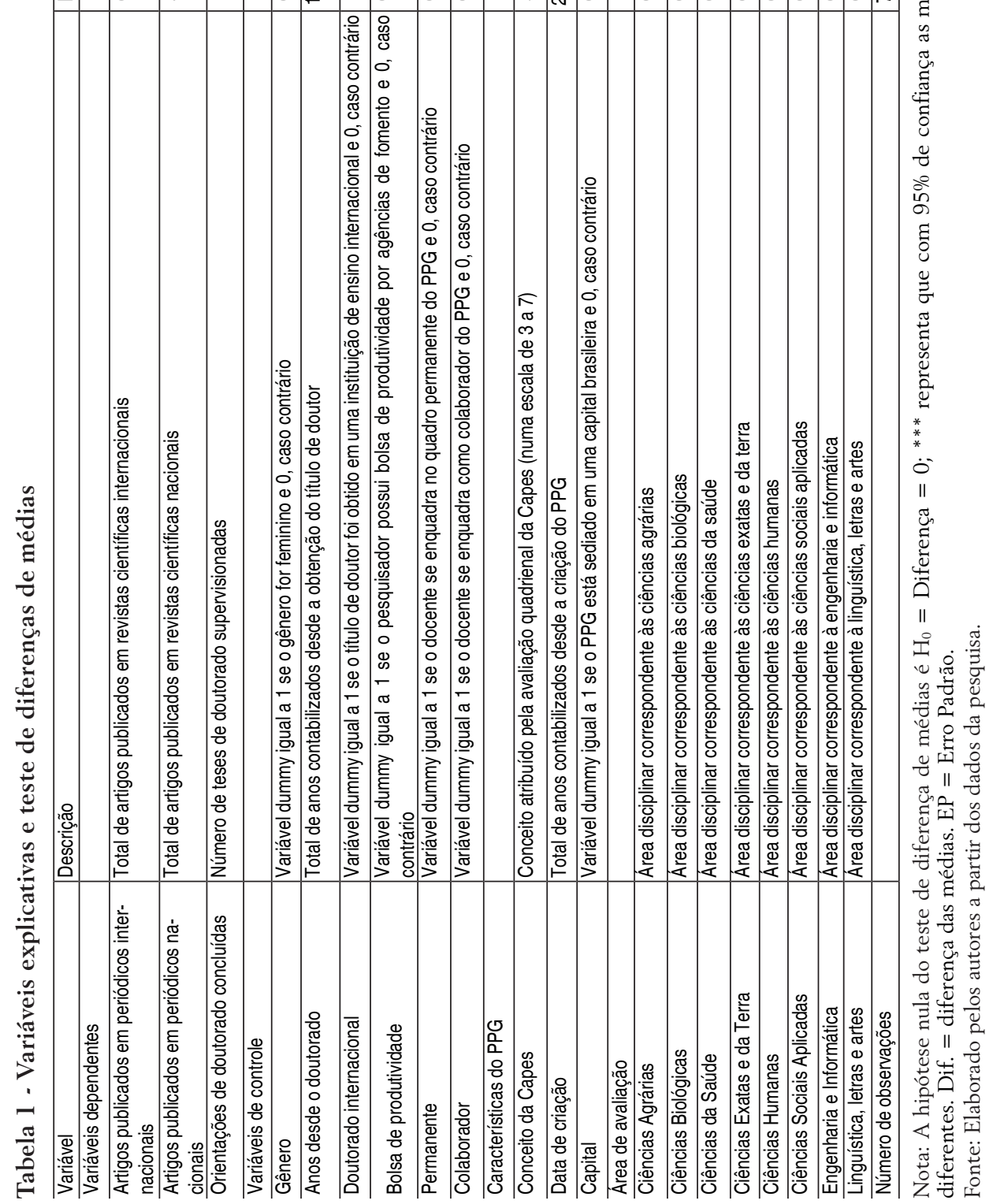




\section{Resultados e discussão}

Essa seção apresenta a análise sobre os docentes com título de doutorado, ao enfatizar as diferenças em produtividade de pesquisa entre acadêmicos que são endógenos e não endógenos por meio do modelo de regressão binomial negativo logit hurdle (NBLH). De modo geral, espera-se que os indivíduos que são endógenos sejam menos produtivos no número de artigos científicos publicados (Sivak e Yudkevich 2015; Horta et al. 2010) e possuam mais alunos de doutorado sob supervisão, ou seja, atuem com maiores cargas horárias de ensino (McGee 1960).

As Tabelas (2, 3 e 4 em Apêndice) apresentam os resultados estimados, sendo para uma melhor interpretação, os coeficientes da regressão foram exponenciados e transformados em razões de probabilidade (OR) na "parte zero do modelo de contagem" e razão de valores (RR) na parte de contagem (não zero) do modelo. As OR refletem a percentagem decrescente $(\mathrm{OR}<1)$ ou aumento percentual $(\mathrm{OR}>1)$ nas probabilidades de possuir ao menos uma publicação, enquanto $R R$ refletem a porcentagem decrescente $(R R<1)$ ou aumento $(R R>1)$ no número esperado de publicações/orientandos para cada unidade de aumento na variável independente, controlando o número dos demais preditores.

A Tabela 2 apresenta os resultados estimados comparando endógenos e não endógenos em relação às publicações internacionais. Primeiro, é possível observar que os docentes não endógenos apresentam uma maior probabilidade de publicarem ao menos um artigo em periódicos internacionais $(\mathrm{OR}=1,122 ; \mathrm{CI} 95 \%=[1,099-1,246] ; \mathrm{p}=0,033)$, embora não haja probabilidade que eles apresentem mais publicações.

Há uma diferença no nível de publicação de acordo com o gênero, em que a parte de contagem (não zero) demonstra que as mulheres são menos propensas a produzir mais de um artigo internacional. Esse resultado é importante, embora esteja fora da questão central deste trabalho. De acordo com Nielsen (2016), as mulheres têm uma menor propensão à colaboração internacional em relação aos colegas masculinos e tendem a publicar mais artigos com autoria única, o que diminui o número de publicações internacionais. $^{5}$

5 Não há consenso na literatura sobre a influência do gênero na produtividade científica, uma vez que os recortes podem ser feitos para diferentes áreas científicas, idade, ranking acadêmico, entre outros fatores. 
Quanto maior o tempo transcorrido desde a titulação, maior o número de publicações, bem como a bolsa de produtividade e a posição como docente permanente no departamento demonstram uma relação positiva com a publicação internacional. O conceito atribuído pela Capes também apresentou uma relação positiva na parte de contagem (não zero), resultado esperado, uma vez que a publicação docente é componente essencial na avaliação quadrienal da agência.

Esses resultados fazem parte de processos de vantagens conjuntas ao longo da carreira, em que mecanismos de feedback entre produtividade e prestígio produzem vantagens cumulativas e efeitos de fortalecimento (CruzCastro e Sanz-Menéndez 2010). Existe uma relação moderada e positiva entre produtividade e prestígio do departamento de contratação que pode ser explicado pela ideia de que cursos melhor conceituados tendem a contratar pessoas mais produtivas (Cole e Cole 1973; Crane 1965).

As melhores instituições acreditam que os próprios estudantes são melhor preparados e têm dificuldade em recrutar pessoas de fora com habilidades e potenciais comparáveis, constituindo um ponto de orgulho para essas universidades e uma continuidade das atividades, identidade institucional e valores (Horta e Yudkevich 2016; Altbach et al. 2015). Os programas considerados excelentes, de conceito 6 e 7, muito provavelmente são os principais fomentadores de mão de obra para outras universidades, já que são referências em suas respectivas áreas.

As capitais brasileiras apresentaram significância negativa na parte de contagem (não zero), tanto para endógenos, como para não endógenos, na publicação de artigos em periódicos nacionais e internacionais. Entretanto, a parte zero do modelo de contagem positiva implica que os pesquisadores endógenos $(\mathrm{OR}=1,174$; CI 95\% = [1,084 - 1,271]; $\mathrm{p}=0,000)$ e não endógenos $(\mathrm{OR}=1,142 ; \mathrm{CI} 95 \%=[1,054-1,238] ; \mathrm{p}=0,001)$ situados nas capitais apresentam uma maior probabilidade de publicarem ao menos um artigo em periódicos nacionais.

A menor publicação de artigos internacionais é um resultado consistente com a concentração de revistas nacionais bem-conceituadas nos grandes centros e com as dificuldades de adequar a produção a periódicos internacionais. Sidone et al. (2017) destacam que, países em desenvolvimento possuem uma concentração de atividades em torno da localização das uni- 
versidades públicas. No Brasil, há uma semelhança entre universidades nas capitais e no interior. ${ }^{6}$

Adaptando uma expressão utilizada para os cientistas políticos, Amorim Neto e Santos (2015) chamaram a atenção para um certo paroquialismo da produção científica nomeando quatro aspectos principais que suportam essa denominação: i) o caráter residual de trabalhos em colaboração com pesquisadores estrangeiros; ii) o conteúdo da produção, voltado para temas específicos da realidade nacional com poucas comparações práticas; iii) o idioma, tanto o inglês, língua franca da comunidade científica internacional, como o espanhol se direcionado para revistas latino-americanas; e iv) os periódicos brasileiros utilizados para a divulgação.

A parte de contagem (não zero) do modelo não significativa sobre a publicação de periódicos internacionais chama atenção para a falta de incentivo voltada para esse fim, uma vez que é plausível pressupor que a internacionalização da produção demande investimentos consideráveis e contínuos por parte dos docentes (Madeira e Marenco 2016). Dessa forma, pode-se considerar que a internacionalização da produção ocorre no médio e longo prazos e com docentes de carreiras mais longevas e estruturadas como referências nas áreas de atuação, o que foi retratado nos resultados estimados pelo tempo que o indivíduo é doutor e a posição de docente.

Segundo Madeira e Marenco (2016), uma vez que a produção acadêmica internacional demanda um investimento, algumas iniciativas governamentais podem ser voltadas para intensificar o processo de internacionalização. O V Plano Nacional de Pós-Graduação (2011-2020), atualmente em vigor, traça estratégias de estímulo à formação de redes de pesquisa por meio de parcerias, nacionais e internacionais, a fim de "nivelar" as atividades científicas a padrões de exigência mais elevados.

Nesse sentido, a busca pela mobilidade internacional - por meio do envio de estudantes ao exterior para cursarem doutorado - e o estímulo à vinda de estudantes estrangeiros, promovem um salto de qualidade e evitam a endogenia na academia (Brasil 2010). Conforme mencionado, esse projeto precisa de tempo para tornar-se efetivo, fato este que pode ser corroborado com o parâmetro estimado para a conclusão do doutorado em outro

6 De acordo com dados divulgados pelo Instituto de Estudos Pesquisas Educacionais Anísio Teixeira (Inep) do Censo do Ensino Superior, em 2018, das 107 universidades públicas brasileiras, 58 (54,2\%) estão localizadas no interior e $49(45,8 \%)$ em capitais. 
país, que não garante a possibilidade de publicar um artigo em periódicos internacionais $(\mathrm{OR}=0,670 ; \mathrm{CI} 95 \%=[0,579-0,776] ; \mathrm{p}=0,000)$.

Em relação à publicação de periódicos nacionais, a Tabela 3 demonstra que os não endógenos publicam mais artigos em relação aos endógenos. A parte de contagem (não zero) significativa e positiva (RR = 1,079; CI 95\% $=[0,0194-0,133] ; \mathrm{p}=0,008)$ indica que os docentes não endógenos possuem maior probabilidade de publicarem artigos em periódicos nacionais. Esse resultado vai contra a premissa que locais com maior endogenia podem ser mais voltados para periódicos nacionais (Sivak e Yudkevich 2015; Birnbaum 2005; Velho e Krige 1984).

Sivak e Yudkevich (2009) alcançaram resultados semelhantes, embora as revistas nacionais fossem diferenciadas. De acordo com os autores, os não endógenos têm mais publicações nas revistas nacionais de maior qualidade enquanto os endógenos estão voltados para revistas da própria instituição, que, em geral, possuem menor qualidade. A interpretação desse resultado deve, no entanto, ser feita com cuidado, uma vez que endógenos e não endógenos podem ter, simplesmente, diferentes estratégias de publicação (Horta et al. 2010).

A demanda e o escopo da pesquisa exigidos pelas publicações nacionais podem se mostrar menos rigorosas, simplificando o processo de publicação, mesmo com a revisão por pares (Horta 2013; Shimbori 1981). Mesmo que garantam e mantenham uma cultura científica nacional ao divulgar contribuições relevantes, as revistas nacionais têm um público-alvo reduzido e, consequentemente, menor impacto e visibilidade das publicações (Kyvik 2003).

O sistema atual de classificação das revistas científicas brasileiras, a Qualis Periódicos, em vigor desde 2001, é um indicador de qualidade desenvolvido pela Capes que define as escolhas e a qualidade das publicações. Essa classificação apresenta inúmeras inconsistências, especialmente ao colocar nos principais estratos ${ }^{7}$ (Al e A2), revistas brasileiras, com menor rigor de avaliação, e revistas internacionais de critérios mais rigorosos. Outra fragilidade desse sistema de classificação é desconsiderar revistas importantes

7 A classificação de periódicos é trienal e subdividida em 49 áreas de avaliação. A atualização da lista de Periódicos do Qualis ocorre anualmente e enquadra os títulos das revistas em estratos indicativos de qualidade, sendo A1, o mais elevado; A2; A3, A4, B1, B2, B3, B4, B5 e C. 
ou reduzir as notas de publicações interdisciplinares ou muito especializadas (Barata 2015).

Muitos periódicos nacionais não possuem destaque nas grandes bases de dados indexadoras internacionais (Silva et al. 2016) e apresentam um fator de impacto muito baixo (Kimura 2010). De acordo com Mugnaini et al. (2014), as revistas nacionais reforçam as diferenças existentes entre as áreas de avaliação e seu grau de internacionalização. Um Qualis mais justo seria viável por meio da descontinuação do fator de impacto, por ser indireto e retratar um desempenho passado, bem como assinalar a presença dos indexadores (Trzesniak 2016).

Ainda em relação aos resultados apresentados na Tabela 3, quanto maior o tempo transcorrido da titulação do docente e da criação do curso, menor a quantidade de publicações nacionais (contagem), o que pode indicar uma estratégia voltada para periódicos internacionais ou um processo acumulado de aprendizagem, especialmente para pesquisadores que não possuem formações no exterior. Mesmo que classificadas nos mesmos estratos de avaliação, as publicações internacionais são utilizadas nos rankings universitários globais, possuem maior visibilidade e evidenciam melhor qualidade de pesquisa (Inanc e Tuncer 2011).

Quando feita a diferenciação por área de avaliação, as ciências agrárias e da saúde correspondem positivamente tanto nos periódicos nacionais como nos internacionais. Essa constatação vai ao encontro do relatório "A pesquisa no Brasil: Promovendo a excelência" desenvolvido para a Capes (2019), em que a pesquisa brasileira apresenta um perfil bem definido. De modo geral, há uma certa concentração das atividades, recursos e excelência em determinadas áreas do conhecimento.

Todavia, trabalhos que contemplam as áreas de ciências humanas, sociais aplicadas, linguística, letras e artes têm menores probabilidades de serem publicados. Esse resultado confirma que existe uma cultura nacional de publicações diversificada de acordo com a área (Cruz-Castro e SanzMenéndez 2010), embora seja pertinente a análise da produção científica específica em relação à sua dinâmica de difusão (Faria et al. 2010).

As ciências biológicas, exatas e da saúde normalmente possuem melhor comunicação com a comunidade científica, especialmente por meio de artigos com estruturas preestabelecidas, o que torna a linguagem mais direcionada 
e com facilidade de adaptação para o inglês. Já as artes, humanidades e ciências sociais têm gêneros de publicações ${ }^{8}$ mais variados e diferentes linguagens, publicadas principalmente no formato de livros e trabalhos em anais de congressos após longos períodos de pesquisa (Souza et al. 2018).

Considerando apenas o número de publicações, a Figura (1) apresenta o efeito da endogenia na publicação de artigos em periódicos nacionais e internacionais.

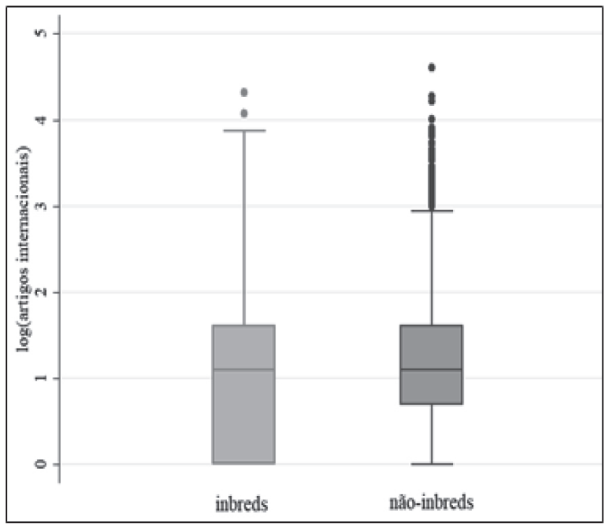

(a)

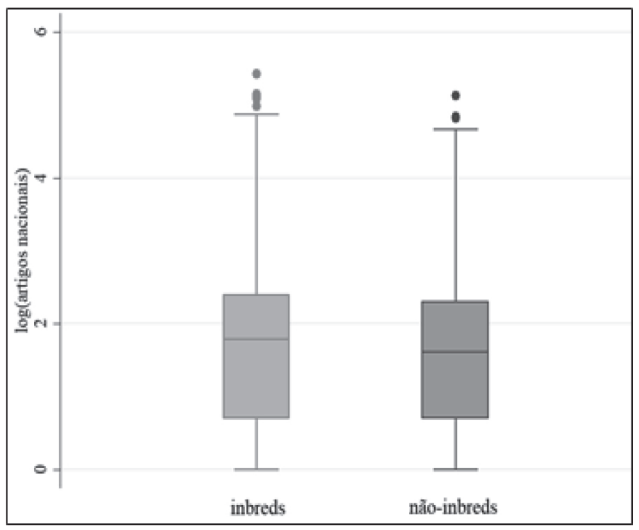

(b)

Figura 1 - Efeito da endogenia na probabilidade de publicações de artigos em periódicos nacionais (a) e internacionais (b)

Fonte: Elaborado pelos autores a partir dos dados da pesquisa.

A parte (a) demonstra que os não endógenos possuem maior dispersão nas publicações internacionais, bem como uma maior incidência de outliers; já os endógenos possuem concentração de publicações no quartil inferior. $\mathrm{Na}$ parte (b), que retrata os periódicos nacionais, os endógenos e não endógenos têm concentrações semelhantes, embora haja uma maior dispersão e presença de outliers para os endógenos.

Por fim, em relação ao número de teses orientadas - variável que combina elementos de ensino e pesquisa - os resultados apresentados na Tabela 4 demonstram resultados significativos na parte zero do modelo de

8 De acordo com Fox (2005), os diferentes gêneros de publicação científica podem assumir a forma de artigos em revistas especializadas, artigos em outras publicações, anais de conferências, críticas, respostas e comentários, resumos, livros, capítulos, artigos em jornais, boletins, entre outros. 
contagem. As probabilidades de possuir um orientando contra nenhum é crescente para os docentes que são endógenos (OR = 1,106; CI 95\% = $[1,028-1,191] ; \mathrm{p}=0.007)$ e decrescente para os não endógenos $(\mathrm{OR}=$ $0,864 ; \mathrm{CI} 95 \%=[0,799-0,934] ; \mathrm{p}=0,000)$, indicando que os indivíduos que são endógenos dedicam-se mais a atividades de ensino e administrativas (Gorelova e Yudkevich 2015; Horta 2013; Dutton 1980).

Esse resultado pode ser um indicativo de que esses indivíduos possuam menor prestígio entre os pares e sejam persuadidos a voltar as atenções para a docência. De acordo com McGee (1960), os endógenos recebem menos recompensas institucionais, possuem um período de tempo mais longo antes de serem promovidos e maiores cargas horárias de ensino. É possível que os endógenos com a posição de professor possuam ligeiramente mais publicações, comparadas aos indivíduos que não são endógenos, entretanto, nas posições mais elevadas, como a de professor permanente, as publicações são maiores para os não endógenos (Shen et al. 2015).

O tempo transcorrido desde o doutorado e o conceito da Capes apresentam relações positivas com a orientação, em que os professores há mais tempo em cursos de maior prestígio permanecem com mais alunos tutelados, o que pode gerar mais publicações derivadas dos trabalhos de conclusão. Os professores mais antigos, por muitas vezes, não possuem maiores incentivos para pesquisa e publicação, como melhorias salariais, voltando suas atividades para o ensino e orientação (Yudkevich 2014).

Eisenberg e Wells (2000) consideram que os alunos endógenos passam por um período voltado ao ensino antes de se dedicarem à pesquisa, o que faz parte de um processo de treinamento relacionado à continuidade das atividades desenvolvidas durante o doutorado. É possível ainda que os próprios alunos sejam recrutados por seu evidente potencial de ensino ou didática, bem como pela perpetuação da política universitária, valores ou diretrizes estabelecidas pelos departamentos (Sivak e Yudkevich 2015; Rocca 2007).

A conexão formada entre os orientadores e os alunos durante o doutorado é muito forte e tende a prevalecer durante a carreira acadêmica, sendo que muitas vezes os orientadores assumem uma figura paternalista (Horta et al. 2011). É comum que os melhores estudantes de doutorado sejam envolvidos em atividades de ensino e pesquisa na fase inicial de seus estudos, o que garante a permanência no meio acadêmico e restringe oportunidades de emprego no mercado de trabalho externo (Alipova e Lovakov 2018). 
Cummings (1975) afirma que alunos da mesma instituição têm um maior senso de dever para com os professores e orientador, considerando determinadas funções como "débitos a serem pagos" pelo favorecimento ou reconhecimento das suas capacidades no processo de seleção. As funções atribuídas podem ser uma maior carga de atividades administrativas e de ensino, bem como o prosseguimento das atividades e temas de pesquisa do orientador.

A Figura (2) apresenta o efeito da endogenia nas orientações de doutorado. É possível observar que a distribuição é muito semelhante, embora não endógenos apresentem mais outliers, provavelmente decorrente do maior número de orientandos de pesquisadores que são referência em suas respectivas áreas de atuação.

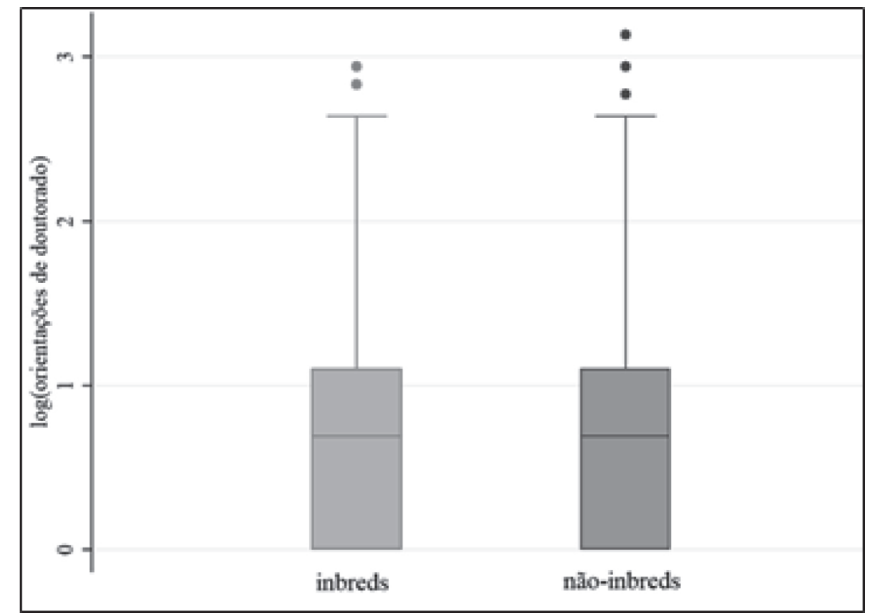

Figura 2 - Efeito da endogenia na probabilidade de orientar teses de doutorado Fonte: Elaborado pelos autores a partir dos dados da pesquisa.

Em suma, os resultados empíricos demonstraram que os não endógenos têm maiores chances de possuir ao menos um artigo internacional e maiores probabilidades de publicações nacionais. Essa diferença pode não ser forte ou robusta o suficiente ao ponto de dizermos categoricamente que os não endógenos são mais produtivos (Smyth e Mishra 2014; Eelss e Cleveland 1935). Em relação a teses de doutorado orientadas, o resultado foi favorável para os professores formados pela mesma instituição, o que 
indica uma maior carga horária em atividades de ensino para esses profissionais (Horta 2013).

De modo geral, não há consenso sobre as causas da endogenia acadêmica, por sua natureza complexa com muitos fatores inter-relacionados (Altbach et al. 2015; Yudkevich et al. 2015). Existente em pequenos e grandes sistemas, pode ter se perpetuado por uma série de motivos, como a falta de atratividade financeira e social ao perseguir uma carreira acadêmica, tradições de imobilidade no mercado de trabalho e sociedade, ênfase no ensino, um mercado de trabalho acadêmico fraco ou inexistente, poucas oportunidades de emprego para doutores, ceticismo nos mecanismos de seleção e contratação, entre outros.

Muitos desses fatores podem ser encontrados em sistemas acadêmicos, como no Brasil e demais países com um sistema educacional caracterizado por estruturas rígidas, poucos incentivos de carreira e "recompensas" por melhor performance. As universidades brasileiras possuem um conjunto de estruturas relativamente pouco responsivas aos desafios sociais, o que as torna permeáveis a altos níveis de endogenia (Alipova e Lovakov 2018; Horta et al. 2011).

No Brasil, os salários são consistentes entre as instituições, ou seja, a ausência de incentivos econômicos limita a mobilidade; soma-se a isso a distribuição desigual de universidades pelo país (Horta e Yudkevich 2016). Ademais, o mercado acadêmico brasileiro pode ser considerado pouco desenvolvido, dado que apresenta baixa mobilidade entre universidades e salários uniformemente definidos pelo Estado. Nesse contexto, as condições de trabalho entre as universidades públicas são homogêneas (Tavares et al. 2015; Horta 2013).

Cardoso (2011) ressalta que a formação e o crescimento do mercado de trabalho acadêmico no Brasil ocorreram com certo predomínio de determinadas áreas do conhecimento em algumas regiões do país - notadamente Sul e Sudeste - como resultado da hegemonia e influência de determinados grupos de interesse na comunidade acadêmica. Esse conjunto de características são, muito provavelmente, um legado oriundo da implantação da pós-graduação brasileira no período militar. 
Embora seja contraditório que o regime militar tenha elegido como prioridade a formação de pesquisadores e docentes, foram forjadas alianças tácitas entre militares e acadêmicos para a definição de políticas educacionais de modernização e financiamento (Hostins 2006). A implantação do curso de pós-graduação no Brasil ocorreu com a necessidade de formar profissionais para atender as atividades de ensino e pesquisa no país como alternativa doméstica e mais acessível, em termos de custos, de qualificar os professores das universidades federais (Balbachevsky 2005).

As universidades selecionadas deste trabalho apresentaram um nível de endogenia consideravelmente elevado (41\%). Ao completar, em média, 62 anos de existência, as universidades estão em um estágio inicial de desenvolvimento, precisamente o que Horta et al. (2011) apontaram como favorecedor da endogenia acadêmica. De certa forma, o mercado acadêmico brasileiro está em um período de transição e a endogenia acadêmica pode ajudar as universidades a contratar os melhores candidatos (frequentemente seus próprios estudantes) com menores riscos na situação e assimetria de informações.

O sistema brasileiro ainda não está desenvolvido ao ponto de atender as demandas da sociedade para graus mais elevados de formação, sendo que a criação de cursos ainda não possui a capacidade de impactar o mercado de trabalho ou promover efeitos regionais e sociais (Cirani et al. 2015). O mercado de trabalho para profissionais com alto nível de especialização no Brasil precisa de tempo para se consolidar, processo que será bem sucedido se as políticas praticadas contarem com a adesão da comunidade científica (Cardoso 2011).

A endogenia pode ser vista, inclusive, como um processo de mercado de trabalho interno, favorecendo promoções de professores para altos cargos de administração (Faria e Mixon Júnior 2020). Essa prática pode ser um indicativo da rigidez das estruturas de governança e gestão nas instituições de ensino, além de reforçar a cultura disciplinar e o descompasso entre incentivos de publicações e diretrizes institucionais (Faria e Mixon Júnior 2020; Cardoso et al. 2016).

A pós-graduação no Brasil, cujo quadro atual é altamente dependente de subsídios estatais e tem uma orientação predominantemente acadêmica, que desconsidera sinalizações externas, como as demandas do mercado, precisa de uma mudança de formato (Balbachevsky 2005). Conforme o 
mercado de trabalho acadêmico apresentar uma evolução, práticas como a endogenia tendem a diminuir, sendo que os doutores serão absorvidos como profissionais altamente capacitados para exercer atividades além do ensino e pesquisa nas instituições de ensino superior.

De modo geral, pelos resultados estimados, não podemos afirmar que endogenia é uma prática prejudicial, por conseguinte, não deve ser endereçada como um tema prioritário no sistema de educação brasileiro. A ausência de diferenças na produtividade de pesquisa entre endógenos e não endógenos pode ser derivada de que, em sistemas acadêmicos fechados, as publicações podem não ser o maior critério de comparação. Além disso, é importante considerar as demais práticas profissionais, como diferentes estratégias e culturas de publicação (Alipova e Lovakov 2018; Horta et al. 2010).

\section{Considerações finais}

Este artigo analisa a endogenia acadêmica nas universidades públicas brasileiras e se essa prática, ainda inexplorada para a realidade do Brasil, impacta na produtividade científica dos docentes. Por meio da compilação do número de artigos publicados em periódicos nacionais e internacionais, bem como o número dos alunos de doutorado sob supervisão, nos últimos três anos, dos docentes que atuam em grupos de pesquisa, foi empregado um modelo binomial negativo logit hurdle.

Sob essa ótica, este trabalho explora a formação do corpo docente brasileiro, sua mobilidade profissional e o seu desempenho em pesquisa. Vale destacar que esses achados devem ser interpretados no contexto de características organizacionais e institucionais do sistema acadêmico brasileiro que promovem o desenvolvimento de mercados de trabalho internos de pesquisa. De certo modo, o mercado de trabalho acadêmico é pouco desenvolvido, logo, é possível afirmar que tem passado por um sistema de transição.

Para a análise dos periódicos publicados em revistas internacionais, os indivíduos que não são endógenos apresentaram uma probabilidade maior de terem ao menos um artigo publicado, mas não muitos, o que denota 
certos entraves para acessar esse tipo de periódico. O resultado positivo nas revistas nacionais para os não endógenos mostra que sim, nesse caso, eles são mais produtivos que os endógenos, embora algumas ressalvas devam ser feitas, especialmente em relação ao paroquialismo da produção científica brasileira.

As revistas internacionais estão, muitas vezes, nos mesmos estratos na classificação Qualis, o que, somado as dificuldades em adaptar o idioma e o formato para um periódico internacional de critérios mais rigorosos acaba por diminuir o interesse dos pesquisadores. Uma produção significativa em determinadas áreas evidencia as potencialidades da pesquisa no Brasil, bem como as diferentes culturas disciplinares e padrões de publicação, que podem assumir formas diversas de artigos científicos.

Em relação ao número de teses orientadas, os resultados demonstram resultados significativos na parte zero do modelo de contagem, positivos para endógenos e negativos para não endógenos, indicativo de que os endógenos atuem com maior carga horária de ensino, o que vai ao encontro da literatura. As universidades podem ter preferências em contratar os próprios alunos para atividades de ensino ao reconhecer seu potencial e dar continuidade às diretrizes dos departamentos.

Algumas características do mercado acadêmico brasileiro favorecem a endogenia, como salários homogêneos entre as universidades públicas, a autonomia das instituições/departamentos para estabelecer critérios de seleção dos concursos e a concentração de universidades em alguns estados no território nacional. De certa forma, o mercado acadêmico brasileiro está em um período de transição, após sua implantação durante o regime militar, e a endogenia pode ser favorável nessa transição ao ajudar as universidades a contratar os melhores candidatos com menores riscos e assimetria de informações.

Por fim, destaca-se que a pesquisa apresenta uma série de limitações, entre as quais pode-se citar que a qualidade das publicações não pode ser auferida pela quantidade de trabalhos e determinadas áreas possuem um perfil notadamente diferenciado de difusão, que pode ocorrer por meio de livros ou artigos em anais de congresso, por exemplo. Outra limitação é a análise de docentes que são líderes de grupos de pesquisa poder viesar o número de publicações em função da seletividade da amostra. 
Cumpre destacar, além do ineditismo, que estudos como este têm fundamental importância na identificação das características e potencialidades da pesquisa e mercado de trabalho acadêmico brasileiro. A inserção de doutores fora do meio acadêmico é extremamente restritiva em função de sua formação predominantemente voltada para a pesquisa e das exigências dos programas e agências de fomento, que demandam dedicação exclusiva. A falta de experiência, aliada à falta de oportunidades e pouca valorização do mercado desses profissionais contribui para que os doutores continuem, majoritariamente, atuando em instituições de ensino superior.

\section{Referências}

Alipova, O. e Lovakov, A. 2018. "Academic inbreeding and publication activities of Russian faculty". Tertiary Education and Management 24, no.1: 66-82.

Altbach, P.G., Yudkevich, M. e Rumbley, L.E. 2015. “Academic inbreeding: local challenge, global problem”. Asia Pacific Education Review 16, no.3: 317-330.

Amorim Neto, O. e Santos, F. 2015. "La ciencia política en Brasil en la última década: la nacionalización y la lenta superación del parroquialismo". Revista de Ciencia Política 35, no.1: 19-31.

Balbachevsky, E. 2005. “A pós-graduação no Brasil: novos desafios para uma política bem-sucedida”. In: Brock, C. e Schwartzman, S. (Orgs.) Os desafios da educação no Brasil. Rio de Janeiro: Nova Fronteira, 2005.

Barata, G. 2015. "Em revisão: o impacto da produção científica brasileira para o Brasil”. Ciência e Cultura 67 , no.4: 6-8.

Barbosa, E.T., Lunardi, M.A., Bizatto, L.S. e Biavatti, V.T. 2018. "Relação entre Endogenia e a Avaliação CAPES dos Programas de Pós-Graduação em Ciências Contábeis no Brasil”. Revista de Educação e Pesquisa em Contabilidade 12, no. 2 :169-185.

Bazzan, A.L., e Argenta, V.F. 2011. "Network of collaboration among PC members of Brazilian computer science conferences". Journal of the Brazilian Computer Society 17 no.2: 133-139.

Berelson, B. 1960. Graduate education in the United States. New York: McGraw-Hill.

Birnbaum, R. 2005. "Professor and Sensei: the construction of faculty roles in the United States and Japan". Higher Education Forum 2 :71-92.

Braga, M.M.S. e Venturini, A.E.J.F. 2013. "Endogenia acadêmica em um programa de pós-graduação em direito". In: Mezzaroba, O., Tavares Neto, J.Q. e Vasconcelos, S.A. (Coords.). Direito, educação, ensino e metodologia jurídicos. Florianópolis: FUNJAB.

Brasil. 2010. Ministério da Educação. Coordenação de Aperfeiçoamento de Pessoal de Nível Superior. Plano Nacional de Pós-Graduação (PNPG) 2011-2020. Brasília: CAPES.

Brasil. 2001. Ministério da Educação. Coordenação de Aperfeiçoamento de Pessoal de Nível Superior. $A$ necessidade de desenvolvimento da pós-graduação profissionalizante e o ajustamento do Sistema de Avaliação às características desse segmento. Brasília: CAPES.

Boschma, R. 2005. "Proximity and innovation: a critical assessment”. Regional studies 39, no.1: 61-74. 
Cameron, A.C. e Trivedi, P.K. 2010. Microeconometrics using Stata: Revised Edition. Stata Press.

Cameron, A.C. e Trivedi, P.K. 1998. Regression Analysis of Count Data. New York: Cambridge University Press.

Cardoso, M.S. 2011. A formação do mercado de trabalho acadêmico no Brasil - 1990 a 2009. 2011. 214 f. Tese (Doutorado em sociologia) - Universidade de Brasília.

Cardoso S., Rosa M.J., e Stensaker, B. 2016. "Why is quality in higher education not achieved? The view of academics". Assess Evaluation on Higher Education 41:950-965.

Cirani, C.B.S., Campanario, M. de A. e Silva, H.H.M. da. 2015. "A evolução do ensino da pós-graduação senso estrito no Brasil: análise exploratória e proposições para pesquisa”. Avaliação-Revista da Avaliação da Educação Superior 20, no.1: 163-187.

Clark, S.A. e Larson, R.F. 1972. "Mobility, Productivity, and Inbreeding at Small Colleges: A Comparative Study". Sociology of Education 45, no.4: 426-434.

Cole, J.R. e Cole, S. 1973. Social Stratification in Science. Chicago: The University of Chicago Press.

Conselho Nacional de Desenvolvimento Científico e Tecnológico. Censo do Diretório de Grupos de Pesquisa (2016-2014). Disponível em: <http://lattes.cnpq.br/web/dgp/censos2>. Acesso em: 15 set. 2019.

Coordenação de Aperfeiçoamento de Pessoal de Nível Superior. Dados Abertos. Disponível em: $<$ https://dadosabertos.capes.gov.br/>. Acesso em: 08 nov. 2019.

Coordenação de Aperfeiçoamento de Pessoal de Nível Superior. Portaria n ${ }^{\circ} 81$, de 3 de junho de 2016. Define as categorias de docentes que compõem os Programas de PósGraduação (PPG's) stricto sensu. 2016. Disponível em< https://www.capes.gov.br/images/stories/download/legislacao/06062016-PORTARIA-N-8-De-3-DE-JUNHO-DE-2016.pdf >: Acesso em: 11 out. 2019.

Coutinho, R.X., Dávila, E.S., Santos, W.M., Rocha, J.B., Souza, D.O., Folmer, V., e Puntel, R.L. 2012. Brazilian scientific production in science education. Scientometrics 92, no.3: 697-710.

Crane, D. 1965. "Scientists at major and minor universities: A study of productivity and recognition". American Sociological Review, 699-714.

Cruz-Castro, L. e Sanz-Menéndez, L. 2010. "Mobility versus job stability: Assessing tenure and productivity outcomes". Research Policy 39, no.1: 27-38.

Cummings, W. K. 1975. "Understanding Behavior in Japan's Academic Marketplace". The Journal of Asian Studies 34, no.2: 313-340.

Dillon, N. 2003. "The postdoctoral system under the spotlight: A European Union Directive regulating fixedterm contracts has focused attention on scientific career structures and could have long-term effects on European science". EMBO Reports 4, no.1: 2-4.

Durieux, V., e Gevenois, P.A. 2010. "Bibliometric Indicators: Quality Measurements Of Scientific Publication". Radiology 255, no.2: 342-351.

Dutton, J.E. 1980. The impact of inbreeding and immobility on the professional role and scholarly performance of academic scientists. In: The annual meeting of the American Educational Research Association, Boston.

Eelss, W.C. e Cleveland, A.C. 1935. "Faculty inbreeding". The Journal of Higher Education 6, no.5: 261-269.

Eisenberg, T. e Wells, M.T. 2000. "Inbreeding in law school hiring: Assessing the performance of faculty hired from within". The Journal of Legal Studies 29, no.1: 369-388.

Faria, J.R., Araujo Júnior, A. F., e Shikida, C.D. 2007. “The Citation Pattern of Brazilian Economists”. Estudos Econômicos 37, no.1: 151-166.

Faria, J.R. e Mixon, F.G. 2020. "The Peter and Dilbert Principles applied to academe". Economics of Governance 21: $115-132$.

Faria, J.R., Wanke, P.F., Ferreira, J.J., e Mixon, F.G. 2018. "Research and innovation in higher education: empirical evidence from research and patenting in Brazil". Scientometrics 116, no.1: 487-504. 
Faria, L.I.L., Gregolin, J.A.R. e Hoffmann, W.A.M. 2010. “Análise da produção científica a partir de publicações em periódicos especializados”. In: FAPESP (Ed.). Indicadores de Ciência, Tecnologia e Inovação em São Paulo 2010. São Paulo: FAPESP.

Fox, M.F. 2005. “Gender, family characteristics, and publication productivity among scientists”. Social Studies of Science 35, no.1: 131-150.

Frenken, K., Hardeman, S., e Hoekman, J. 2009. "Spatial scientometrics: Towards a cumulative research program". Journal of informetrics 3, no.3: 222-232.

Godechot, O. e Louvet, A. 2010. Academic Inbreeding: An Evaluation. Disponível em: < https://booksandideas. net/Academic-Inbreeding-An-Evaluation.html>. Acesso em: 07 set. 2019.

Gorelova, O. e Lovakov, A. 2016. "Academic inbreeding and research productivity of Russian faculty members". Higher School of Economics Research Paper, n. wpbrp32/edu.

Gorelova, O. e Yudkevich, M. 2015. “Academic inbreeding: State of the literature”. In: Yudkevich, M., Altbach, P.G. e Rumbley, L.E. (Eds.), Academic inbreeding and mobility in higher education: Global perspectives. New York: Palgrave Macmillan.

Haddad, E.A., Mena-Chalco, J.P., e Sidone, O. 2017. "Produção científica e redes de colaboração dos docentes vinculados aos programas de pós-graduação em Economia no Brasil”. Estudos Econômicos 47, no.4: 617-679.

Hargens, L.L. e Farr, G.M. 1973. “An examination of recent hypotheses about institutional inbreeding”. American Journal of Sociology 78, no.6: 1381-1402.

Hilbe, J.M. 2011. Negative binomial regression. Cambridge University Press.

Hirsch, J.E. 2005. “An index to quantify an individual's scientific research output". Proceedings of the National academy of Sciences 102, no.46: 16569-16572.

Hollingshead, A.B. 1938. "Ingroup membership and academic selection". American Sociological Review 3, no.6: 826-833.

Horta, H. 2013. "Deepening our understanding of academic inbreeding effects on research information exchange and scientific output: new insights for academic based research". Higher Education 65, no.4: 487-510.

Horta, H., Sato, M., Yonezawa, A. 2011. “Academic inbreeding: Exploring its characteristics and rationale in Japanese universities using a qualitative perspective". Asia Pacific Education Review 12, no.1: 35-44.

Horta, H., Veloso, F. e Grediaga, R. 2010. "Navel gazing: Academic inbreeding and scientific productivity". Management Science 56, no.3: 414-429.

Horta, H. e Yudkevich, M. 2016. "The role of academic inbreeding in developing higher education systems: Challenges and possible solutions". Technological Forecasting and Social Change 113: 363-372.

Horta, J.S.B. e Moraes, M.C.M. de. 2005. “O sistema CAPES de avaliação da pós-graduação: da área de educação à grande área de ciências humanas”. Revista Brasileira de Educação 30, no.4: 95-116.

Hostins, R.C.L. 2006. “Os Planos Nacionais de Pós-Graduação (PNPG) e suas repercussões na Pós-graduação brasileira”. Perspectiva 24, no.1: 133-160.

Inanc, O. e Tuncer, O. 2011. "The effect of academic inbreeding on scientific effectiveness". Scientometrics 88, no.3: 885-898.

Kimura, E.T. 2010. “O dilema das revistas científicas brasileiras na divulgação da produção científica nacional". Arquivos Brasileiros de Endocrinologia \& Metabologia 54, no.1: 1-2.

Kyvik, S. 2003. "Changing trends in publishing behaviour among university faculty, 1980-2000". Scientometrics 58, no.1: $35-48$.

Laender, A.H., Lucena, C.J., Maldonado, J.C., Silva, E.S., e Ziviani, N. 2008. “Assessing the research and education quality of the top Brazilian Computer Science graduate programs". ACM SIGCSE Bulletin 40, no.2: $135-145$.

Lafferty, H.M. 1964. “Of time and the teachers colleges in Texas”. Peabody Journal of Education 42, no.1: 14-22.

Loeys, T., Moerkerke, B., Smet, O. de e Buysse, A. 2012. "The analysis of zero-inflated count data: Beyond zero-inflated Poisson regression”. British Journal of Mathematical and Statistical Psychology 65, no.1: 163-180. 
Madeira, R.M. e Marenco, A. 2016. "The challenges of internationalization: mapping dynamics and paths of international flow". Revista Brasileira de Ciência Política 19: 47-74.

Martins, C.B.A. 2009. "Reforma universitária de 1968 e a abertura para o ensino superior privado no Brasil". Educação \& sociedade 30, no.106: 15-35.

Mcgee, R. 1960. "The function of institutional inbreeding”. The American Journal of Sociology, 65, no.5: 483-488.

Mcneely, J.H. 1932. Faculty inbreeding in land-grant Colleges and Universities. Washington: Office of Education.

Mena-Chalco, J.P., Digiampietri, L.A., Lopes, F.M., e Cesar, R.M. 2014. "Brazilian bibliometric coauthorship networks". Journal of the Association for Information Science and Technology 65, no.7: 1424-1445.

Mingers, J., e Leydesdorff, L. 2015. "A review of theory and practice in scientometrics". European journal of operational research 246, no.1: 1-19.

Nalimov, V.V., e Mul'chenko, Z.M. 1971. Measurement of science. Study of the development of science as an information process. Washington: Foreign Technology Division.

Nielsen, M.W. 2016. "Gender inequality and research performance: moving beyond individual-meritocratic explanations of academic advancement”. Studies in Higher Education 41, no.11: 2044-2060.

Pan, S. 1993. A study of faculty inbreeding at eleven land-grant universities. Doctoral dissertation, Iowa State University, Ames.

Ponds, R., Oort, F.V., e Frenken, K. 2009. "Innovation, spillovers and university-industry collaboration: an extended knowledge production function approach". Journal of Economic Geography 10, no.2: 231-255.

Ravenscroft, J., Liakata, M., Clare, A., e Duma, D. 2017. "Measuring scientific impact beyond academia: An assessment of existing impact metrics and proposed improvements". PloS one 12, no.3: 1-23.

Reeves, F.W., Henry, N.B., Kelly, F.J., Klein, A.J. e Russel, J.D. 1933. The university faculty. Chicago: The University of Chicago.

Rocca, F.X. 2007. "In Spain, Inbreeding Threatens Academe". Chronicle of Higher Education, 53, no.22: 1-317.

Royal Society. 2011. Knowledge, networks and nations: Global scientific collaboration in the 21st century. London: The Royal Society. Disponível em: $<$ https://royalsociety.org/policy/projects/knowledge-networks-nations/ report/>. Acesso em: 06 jul. 2020.

Sahu, S.R., e Panda, K.C. 2014. "Does the multi-authorship trend influence the quality of an article?" Scientometrics 98, no.3: 2161-2168.

Shen, H., Xu, Z. e Zhang, B. 2015. Faculty Inbreeding in China: Status, Causes and Results.In: Yudkevich, M., Altbach, P.G., e Rumbley, L.E. (Eds.), Academic inbreeding and mobility in higher education: Global perspectives. New York: Palgrave Macmillan, 2015.

Shimbori, M. 1981. “The Japanese academic profession”. Higher Education 10, no.1: 75-87.

Sidone, O.J.G., Haddad, E.A., e Mena-Chalco, J.P. 2017. "Scholarly publication and collaboration in Brazil: The role of geography". Journal of the Association for Information Science and Technology 68, no.1: 243-258.

Sidone, O.J.G., Haddad, E.A., e Mena-Chalco, J.P. 2016. “A ciência nas regiões brasileiras: evolução da produção e das redes de colaboração científica". Transinformação 28, no.1: 15-32.

Silva, D.M., Carvalho, T.O., Amaro, B., e Assis, T.B. 2016. "O retrato situacional das revistas científicas brasileiras". Cadernos BAD 2: 116-124.

Sistema de Informações Georreferenciadas (GEOCAPES). Coordenação de Aperfeiçoamento de Pessoal de Nivel Superior. Disponível em: < https://geocapes.capes.gov.br/geocapes/>. Acesso em: 18 nov. 2019.

Sivak, E. e Yudkevich, M. 2015. “Academic immobility and inbreeding in Russian University sector”. In: Yudkevich, M., Altbach, P.G. e Rumbley, L.E. (Eds.), Academic inbreeding and mobility in higher education: Global perspectives. New York: Palgrave Macmillan.

Sivak, E. e Yudkevich, M. 2009. “Academic Inbreeding: Pro and Contra”. Educational Studies, 1: 170-187. 
Smyth, R. e Mishra, V. 2014. "Academic inbreeding and research productivity and impact in Australian law schools". Scientometrics 98, no.1: 583-618.

Souza, C.D. de; Filippo, D. de e Casado, E.S. 2018. "Crescimento da atividade científica nas universidades federais brasileiras". Revista da Avaliação da Educação Superior 23, no.1: 126-156.

Stephan, P. e M.A., J. 2005. “The increased frequency and duration of the postdoctorate career stage”. American Economic Review 95, no.2: 71-75.

Tavares, O., Cardoso, S., Carvalho, T., Sousa, S.B. e Santiago, R. 2015. "Academic inbreeding in the Portuguese academia”. Higher Education 69, no.6: 991-1006.

Tavares, O., Lança, V. e Amaral, A. 2017. “Academic Inbreeding in Portugal: Does Insularity Play a Role?" Higher Education Policy 30, no.3: 381-399.

Tavares, O., Sin, C. e Lança, V. 2019. "Inbreeding and Research Productivity Among Sociology PhD Holders in Portugal”. Minerva 57: 373-390.

Trzesniak, P. 2016. "Um Qualis em quatro tempos: Histórico e sugestões para administração, ciências contábeis e turismo". Revista Contabilidade \& Finanças 27, no.72: 279-290.

Velho, L. e Krige, J. 1984. "Publication and citation practices of Brazilian agricultural scientists". Social Studies of Science 14, no.1: 45-62.

Vives, J., Losilla, J.M. e Rodrigo, M.F. 2006. "Count data in psychological applied research". Psychological Reports 98, no.3: 821-835.

Wang, M., Wang, Z., e Chen, G. 2019. "Which can better predict the future success of articles? Bibliometric indices or alternative metrics". Scientometrics 119, no.3: 1575-1595.

Web of Science Group. A Pesquisa no Brasil: Promovendo a excelência. 2019. Disponível em: <https://propp. ufms.br/files/2019/09/Pesquisa-no-Brasil.pdf>. Acesso em: 14 nov. 2019.

Wilson, L. 1942. The Academic Man. New York: Oxford University.

Wu, J. 2019. "Infrastructure of Scientometrics: The Big and Network Picture". Journal of Data and Information Science 4, no.4: 1-12.

Wyer, J.C. e Conrad, C.F. 1984. “Institutional inbreeding re-examined”. American Educational Research Journal 21, no.1: 213-225.

Yamanoi, A. 2005. "The academic marketplace in Japan: Inbreeding, grades and organization at research universities". Higher Education 3, no.1: 93-114.

Yudkevich, M. 2014. "The Russian University: recovery and rehabilitation”. Studies in Higher Education 39, no 8:1463-1474.

Yudkevich, M., Altbach, P.G. e Rumbley, L.E. 2015. Academic inbreeding and mobility in higher education: Global perspectives. New York: Palgrave Macmillan.

Zorzetto, R., Razzouk, D., Dubugras, M.T.B., Gerolin, J., Schor, N., Guimarães, J.A. e Mari, J.J. 2006. “The scientific production in health and biological sciences of the top 20 Brazilian universities". Brazilian Journal of Medical and Biological Research 39, no.12: 1513-1520. 


\section{Apêndice}

Tabela A1 - Valores dos critérios de Akaike para os modelos ZIP, ZINB, PLH e NBLH

\begin{tabular}{l|c|c|c|c}
\hline & ZIP & ZINB & PLH & NBLH \\
\hline Artigos internacionais & 124426.6 & 85503.5 & 121928.1 & 85401.8 \\
\hline Artigos nacionais & 99495.9 & 81384.9 & 98660.5 & 78368.9 \\
\hline Orientações de doutorado & 50505.7 & 52697.8 & 48431.3 & 47696.8 \\
\hline
\end{tabular}

Fonte: Elaborado pelos autores a partir dos dados da pesquisa.

Tabela A2 - Universidades selecionadas e regiões

\begin{tabular}{l|l}
\hline \multicolumn{1}{c|}{ Região } & \multicolumn{1}{c}{ Universidade } \\
\hline Centro-Oeste & $\begin{array}{l}\text { Universidade Federal de Goiás (UFG); Universidade Federal do Mato Grosso do Sul (UFMS); Uni- } \\
\text { versidade Federal do Mato Grosso (UFMT); Universidade de Brasilia (UNB); Universidade Esta- } \\
\text { dual do Centro-Oeste (UNICENTRO). }\end{array}$ \\
\hline \multirow{5}{*}{ Nordeste } & $\begin{array}{l}\text { Universidade Estadual da Paraiba (UEPB); Fundação Universidade Federal do Piauí (FUFPI); } \\
\text { Universidade Federal de Alagoas (UFAL); Universidade Federal da Bahia (UFBA); Universidade } \\
\text { Federal do Ceará (UFC); Universidade Federal do Maranhão (UFMA); Universidade Federal da } \\
\text { Paraíba (UFPB); Universidade Federal do Rio Grande do Norte (UFRN); Universidade Federal } \\
\text { Rural de Pernambuco (UFRPE). }\end{array}$ \\
\hline \multirow{5}{*}{ Sudeste } & $\begin{array}{l}\text { Fundação Universidade Federal de Sergipe (FUFSE); Universidade Federal do Amazonas (UFAM); } \\
\text { Universidade Federal do Pará (UFPA); Universidade Federal de Tocantins (UFT). }\end{array}$ \\
\hline & $\begin{array}{l}\text { Universidade Federal do Espírito Santo (UFES); Universidade Federal Fluminense (UFF); Uni- } \\
\text { versidade Federal de Juiz de Fora (UFJF); Universidade Federal de Minas Gerais (UFMG); Uni- } \\
\text { versidade Federal do Rio de Janeiro (UFRJ); Universidade Federal de São Carlos (UFSCAR); } \\
\text { Universidade Federal de São João del-Rei (UFSJ); Universidade Federal de Uberlândia (UFU); } \\
\text { Universidade Federal de Viçosa (UFV); Universidade Estadual Paulista (UNESP); Universidade } \\
\text { Estadual de Campinas (UNICAMP); Universidade de São Paulo (USP). }\end{array}$ \\
\hline \multirow{5}{*}{ Sul } & $\begin{array}{l}\text { Universidade Federal do Rio Grande (FURG); Universidade do Estado de Santa Catarina (UDESC); } \\
\text { Universidade Estadual de Londrina (UEL); Universidade Estadual de Maringá (UEM); Universidade } \\
\text { Estadual de Ponta Grossa (UEPG); Universidade Federal de Pelotas (UFPEL); Universidade Fede- } \\
\text { ral do Paraná (UFPR); Universidade Federal do Rio Grande do Sul (UFRGS); Universidade Federal } \\
\text { de Santa Catarina (UFSC); Universidade Federal de Santa Maria (UFSM). }\end{array}$ \\
\hline
\end{tabular}

Fonte: Elaborado pelos autores a partir dos dados do Geocapes. 
Tabela 2 - Resultados estimados pelo modelo NBLH comparando publicações em periódicos internacionais para docentes endógenos e não endógenos

\begin{tabular}{|c|c|c|c|c|}
\hline & Periódicos internacionais & & riódicos internacion & \\
\hline & Contagem & Zero & Contagem & Zero \\
\hline Gênero & $\begin{array}{c}-0.217^{\star \star \star} \\
(0.0210)\end{array}$ & $\begin{array}{l}0.231^{\star \star \star} \\
(0.0472)\end{array}$ & $\begin{array}{c}-0.218^{\star \star \star} \\
(0.0211)\end{array}$ & $\begin{array}{l}0.217^{\star \star \star} \\
(0.0473)\end{array}$ \\
\hline Anos desde o doutorado & $\begin{array}{l}0.007^{* * *} \\
(0.0012)\end{array}$ & $\begin{array}{c}-0.0006 \\
(0.0028)\end{array}$ & $\begin{array}{c}0.0068^{* \star *} \\
(0.0012)\end{array}$ & $\begin{array}{c}0.0023 \\
(0.0028)\end{array}$ \\
\hline Bolsa de produtividade & $\begin{array}{l}0.806^{* * *} \\
(0.0229)\end{array}$ & $\begin{array}{c}-1.256^{\star \star *} \\
(0.0578)\end{array}$ & $\begin{array}{l}0.806^{\star \star *} \\
(0.0228)\end{array}$ & $\begin{array}{r}-1.257^{* \star *} \\
(0.0580)\end{array}$ \\
\hline Permanente & $\begin{array}{l}0.407^{\star *} \\
(0.1260)\end{array}$ & $\begin{array}{l}-0.392 \\
(0.283)\end{array}$ & $\begin{array}{l}0.400^{\star *} \\
(0.127)\end{array}$ & $\begin{array}{l}-0.480 \\
(0.281)\end{array}$ \\
\hline Colaborador & $\begin{array}{c}0.128 \\
(0.130)\end{array}$ & $\begin{array}{c}0.256 \\
(0.288)\end{array}$ & $\begin{array}{c}0.120 \\
(0.131)\end{array}$ & $\begin{array}{c}0.168 \\
(0.285)\end{array}$ \\
\hline Conceito da Capes & $\begin{array}{l}0.130^{\star \star *} \\
(0.0105)\end{array}$ & $\begin{array}{c}-0.222^{* * *} \\
(0.0246)\end{array}$ & $\begin{array}{l}0.130^{* * *} \\
(0.0106)\end{array}$ & $\begin{array}{l}-0.212^{* \star \star} \\
(0.0246)\end{array}$ \\
\hline Data de criação & $\begin{array}{l}-0.0011 \\
(0.0008)\end{array}$ & $\begin{array}{l}-0.0039^{*} \\
(0.0019)\end{array}$ & $\begin{array}{l}-0.00106 \\
(0.0008)\end{array}$ & $\begin{array}{l}-0.0036 \\
(0.0019)\end{array}$ \\
\hline Capital & $\begin{array}{c}-0.0931^{* * *} \\
(0.0223)\end{array}$ & $\begin{array}{c}-0.0698 \\
(0.0510)\end{array}$ & $\begin{array}{c}-0.0906^{* * *} \\
(0.0223)\end{array}$ & $\begin{array}{l}-0.0343 \\
(0.0514)\end{array}$ \\
\hline Ciências Agrárias & $\begin{array}{l}0.163^{* \star *} \\
(0.0446)\end{array}$ & $\begin{array}{c}-1.766^{\star \star \star} \\
(0.125)\end{array}$ & $\begin{array}{l}0.164^{\star \star *} \\
(0.0446)\end{array}$ & $\begin{array}{r}-1.777^{\star \star \star} \\
(0.125)\end{array}$ \\
\hline Ciências Biológicas & $\begin{array}{l}0.245^{\star \star \star} \\
(0.0443)\end{array}$ & $\begin{array}{c}-2.418^{\star \star \star} \\
(0.169)\end{array}$ & $\begin{array}{l}0.244^{\star \star \star} \\
(0.0443)\end{array}$ & $\begin{array}{r}-2.436^{* \star \star} \\
(0.169)\end{array}$ \\
\hline Ciências da Saúde & $\begin{array}{l}0.570^{\star \star \star} \\
(0.0424)\end{array}$ & $\begin{array}{c}-2.015^{\star \star \star} \\
(0.113)\end{array}$ & $\begin{array}{l}0.569^{\star \star *} \\
(0.0424)\end{array}$ & $\begin{array}{c}-2.030^{\star \star \star} \\
(0.113)\end{array}$ \\
\hline Ciências Exatas e da Terra & $\begin{array}{l}-0.132^{\star \star} \\
(0.0476)\end{array}$ & $\begin{array}{c}-1.071^{\star \star \star} \\
(0.102)\end{array}$ & $\begin{array}{l}-0.132^{\star *} \\
(0.0475)\end{array}$ & $\begin{array}{r}-1.074^{\star \star x} \\
(0.102)\end{array}$ \\
\hline Ciências Humanas & $\begin{array}{c}-1.272^{\star \star \star} \\
(0.0726)\end{array}$ & $\begin{array}{l}1.621^{\star \star \star} \\
(0.0813)\end{array}$ & $\begin{array}{r}-1.272^{\star \star \star} \\
(0.0725)\end{array}$ & $\begin{array}{l}1.609^{\star \star \star} \\
(0.0814)\end{array}$ \\
\hline Ciências Sociais Aplicadas & $\begin{array}{l}-1.374^{\star \star *} \\
(0.0831)\end{array}$ & $\begin{array}{l}1.644^{* \star *} \\
(0.0924)\end{array}$ & $\begin{array}{r}-1.372^{* \star *} \\
(0.0831)\end{array}$ & $\begin{array}{l}1.658^{\star \star \star} \\
(0.0926)\end{array}$ \\
\hline Engenharia e Informática & $\begin{array}{l}-0.291^{\star \star \star} \\
(0.0505)\end{array}$ & $\begin{array}{c}-0.756^{* \star *} \\
(0.106)\end{array}$ & $\begin{array}{c}-0.287^{\star \star \star} \\
(0.0506)\end{array}$ & $\begin{array}{r}-0.729^{* \star *} \\
(0.106)\end{array}$ \\
\hline Linguística, letras e artes & $\begin{array}{c}-2.007^{\star \star \star} \\
(0.131)\end{array}$ & $\begin{array}{l}2.438^{\star \star *} \\
(0.0985)\end{array}$ & $\begin{array}{c}-2.004^{\star \star \star} \\
(0.131)\end{array}$ & $\begin{array}{l}2.448^{\star \star \star} \\
(0.0987)\end{array}$ \\
\hline Doutorado internacional & & & $\begin{array}{l}-0.0329 \\
(0.0304)\end{array}$ & $\begin{array}{c}-0.400^{* \star *} \\
(0.0747)\end{array}$ \\
\hline Endógenos & $\begin{array}{c}0.0307 \\
(0.0233)\end{array}$ & $\begin{array}{c}-0.0121 \\
(0.0509)\end{array}$ & & \\
\hline Não endógenos & & & $\begin{array}{l}-0.0507 \\
(0.0252)\end{array}$ & $\begin{array}{c}0.115^{*} \\
(0.0538)\end{array}$ \\
\hline Log-Likelihood & -42663.57 & & -42647.33 & \\
\hline AIC & 85401.14 & & 85372.66 & \\
\hline $\mathrm{N}$ & 7186 & & 10428 & \\
\hline
\end{tabular}

Nota: Desvio-padrão entre parênteses; ${ }^{*} \mathrm{p}<0.05,{ }^{* *} \mathrm{p}<0.01,{ }^{* * *} \mathrm{p}<0.001$.

Fonte: Elaborado pelos autores a partir dos dados da pesquisa. 
Tabela 3 - Resultados estimados pelo modelo NBLH comparando publicações em periódicos nacionais para docentes endógenos e não endógenos

\begin{tabular}{|c|c|c|c|c|}
\hline & \multicolumn{2}{|c|}{ Periódicos nacionais } & \multicolumn{2}{|c|}{ Periódicos nacionais } \\
\hline & Contagem & Zero & Contagem & Zero \\
\hline Gênero & $\begin{array}{c}-0.0668^{\star \star} \\
(0.0252)\end{array}$ & $\begin{array}{c}-0.0306 \\
(0.0385)\end{array}$ & $\begin{array}{c}-0.0703^{* \star} \\
(0.0253)\end{array}$ & $\begin{array}{c}-0.0157 \\
(0.0386)\end{array}$ \\
\hline Anos desde o doutorado & $\begin{array}{c}-0.00807^{\star \star \star} \\
(0.0016)\end{array}$ & $\begin{array}{c}0.0132^{\star \star \star} \\
(0.0021)\end{array}$ & $\begin{array}{c}-0.0073^{\star \star \star} \\
(0.0253)\end{array}$ & $\begin{array}{c}0.0110^{\star \star \star} \\
(0.0021)\end{array}$ \\
\hline Bolsa de produtividade & $\begin{array}{l}0.397^{\star * *} \\
(0.0288)\end{array}$ & $\begin{array}{c}-0.628^{* * *} \\
(0.0437)\end{array}$ & $\begin{array}{l}0.398^{* * *} \\
(0.0287)\end{array}$ & $\begin{array}{c}-0.634^{* * *} \\
(0.0438)\end{array}$ \\
\hline Permanente & $\begin{array}{l}0.360^{*} \\
(0.157)\end{array}$ & $\begin{array}{c}-0.579^{* *} \\
(0.221)\end{array}$ & $\begin{array}{l}0.343^{*} \\
(0.157)\end{array}$ & $\begin{array}{l}-0.518^{*} \\
(0.219)\end{array}$ \\
\hline Colaborador & $\begin{array}{l}0.0743 \\
(0.160)\end{array}$ & $\begin{array}{l}0.0501 \\
(0.223)\end{array}$ & $\begin{array}{l}0.0567 \\
(0.160)\end{array}$ & $\begin{array}{c}0.114 \\
(0.222)\end{array}$ \\
\hline Conceito da Capes & $\begin{array}{l}-0.0171 \\
(0.0124)\end{array}$ & $\begin{array}{c}-0.0142 \\
(0.0187)\end{array}$ & $\begin{array}{l}-0.0139 \\
(0.0124)\end{array}$ & $\begin{array}{l}-0.0244 \\
(0.0187)\end{array}$ \\
\hline Data de criação & $\begin{array}{c}-0.0041^{* * *} \\
(0.0011)\end{array}$ & $\begin{array}{c}0.0076^{* * *} \\
(0.0015)\end{array}$ & $\begin{array}{c}-0.0040^{\star \star *} \\
(0.0011)\end{array}$ & $\begin{array}{l}0.0071^{\star \star *} \\
(0.00151)\end{array}$ \\
\hline Capital & $\begin{array}{c}-0.0916^{\star \star \star} \\
(0.0256)\end{array}$ & $\begin{array}{l}0.161^{* * *} \\
(0.0406)\end{array}$ & $\begin{array}{c}-0.0824^{* *} \\
(0.0256)\end{array}$ & $\begin{array}{l}0.133^{\star *} \\
(0.0409)\end{array}$ \\
\hline Ciências Agrárias & $\begin{array}{l}0.302^{* * *} \\
(0.0551)\end{array}$ & $\begin{array}{c}-0.550^{* \star *} \\
(0.0995)\end{array}$ & $\begin{array}{l}0.301^{\star * *} \\
(0.0551)\end{array}$ & $\begin{array}{c}-0.545^{* * *} \\
(0.0997)\end{array}$ \\
\hline Ciências Biológicas & $\begin{array}{l}-0.581^{* * *} \\
(0.0622)\end{array}$ & $\begin{array}{l}0.685^{\star \star *} \\
(0.0883)\end{array}$ & $\begin{array}{c}-0.585^{* * *} \\
(0.0621)\end{array}$ & $\begin{array}{l}0.705^{\star \star *} \\
(0.0887)\end{array}$ \\
\hline Ciências da Saúde & $\begin{array}{l}0.195^{\star \star *} \\
(0.0541)\end{array}$ & $\begin{array}{c}-0.0573 \\
(0.0848)\end{array}$ & $\begin{array}{l}0.191^{\star \star *} \\
(0.0539)\end{array}$ & $\begin{array}{c}-0.0443 \\
(0.0850)\end{array}$ \\
\hline Ciências Exatas e da Terra & $\begin{array}{c}-0.830^{\star \star \star} \\
(0.0628)\end{array}$ & $\begin{array}{l}1.129^{\star \star \star} \\
(0.0842)\end{array}$ & $\begin{array}{c}-0.831^{\star \star \star} \\
(0.0625)\end{array}$ & $\begin{array}{l}1.133^{\star \star *} \\
(0.0844)\end{array}$ \\
\hline Ciências Humanas & $\begin{array}{l}-0.0944 \\
(0.0525)\end{array}$ & $\begin{array}{c}-0.434^{* \star *} \\
(0.0890)\end{array}$ & $\begin{array}{l}-0.0994 \\
(0.0523)\end{array}$ & $\begin{array}{l}-0.418^{* \star *} \\
(0.0892)\end{array}$ \\
\hline Ciências Sociais Aplicadas & $\begin{array}{c}0.0414 \\
(0.0588)\end{array}$ & $\begin{array}{c}-0.352^{* \star *} \\
(0.103)\end{array}$ & $\begin{array}{c}0.0430 \\
(0.0585)\end{array}$ & $\begin{array}{c}-0.360^{* * *} \\
(0.103)\end{array}$ \\
\hline Engenharia e Informática & $\begin{array}{l}-0.500^{* \star *} \\
(0.0698)\end{array}$ & $\begin{array}{l}0.706^{* * *} \\
(0.0903)\end{array}$ & $\begin{array}{c}-0.490^{* * *} \\
(0.0696)\end{array}$ & $\begin{array}{l}0.682^{* * *} \\
(0.0906)\end{array}$ \\
\hline Linguística, letras e artes & $\begin{array}{c}-0.415^{\star \star \star} \\
(0.0588)\end{array}$ & $\begin{array}{c}0.0457 \\
(0.0976)\end{array}$ & $\begin{array}{c}-0.416^{\star \star \star} \\
(0.0586)\end{array}$ & $\begin{array}{c}0.0449 \\
(0.0977)\end{array}$ \\
\hline Doutorado internacional & & & $\begin{array}{l}-0.120^{* *} \\
(0.0391)\end{array}$ & $\begin{array}{l}0.336^{* * *} \\
(0.0549)\end{array}$ \\
\hline Endógenos & $\begin{array}{l}-0.0468 \\
(0.0275)\end{array}$ & $\begin{array}{c}0.0456 \\
(0.0396)\end{array}$ & & \\
\hline Não endógenos & & & $\begin{array}{l}0.0762^{\star *} \\
(0.0290)\end{array}$ & $\begin{array}{l}-0.151^{\star \star \star} \\
(0.0432)\end{array}$ \\
\hline Log-Likelihood & & & & \\
\hline AIC & & & & \\
\hline $\mathrm{N}$ & & & & \\
\hline
\end{tabular}

Nota: Desvio-padrão entre parênteses; ${ }^{*} \mathrm{p}<0.05,{ }^{* *} \mathrm{p}<0.01,{ }^{* * *} \mathrm{p}<0.001$.

Fonte: Elaborado pelos autores a partir dos dados da pesquisa. 
Tabela 4 - Resultados estimados pelo modelo NBLH comparando o número de orientações de doutorado para docentes endógenos e não endógenos

\begin{tabular}{|c|c|c|c|c|}
\hline & \multicolumn{2}{|c|}{ Orientações de doutorado } & \multicolumn{2}{|c|}{ Orientações de doutorado } \\
\hline & Contagem & Zero & Contagem & Zero \\
\hline Gênero & $\begin{array}{l}-0.0254 \\
(0.0238)\end{array}$ & $\begin{array}{c}-0.0895^{\star} \\
(0.0355)\end{array}$ & $\begin{array}{l}-0.0266 \\
(0.0239)\end{array}$ & $\begin{array}{l}-0.0831^{*} \\
(0.0356)\end{array}$ \\
\hline Anos desde o doutorado & $\begin{array}{l}0.0121^{* * *} \\
(0.0014)\end{array}$ & $\begin{array}{l}-0.0802^{*} \\
(0.0025)\end{array}$ & $\begin{array}{c}0.0123^{* * *} \\
(0.0015)\end{array}$ & $\begin{array}{c}-0.0814^{* * *} \\
(0.0025)\end{array}$ \\
\hline Bolsa de produtividade & $\begin{array}{l}0.456^{\star \star \star} \\
(0.0246)\end{array}$ & $\begin{array}{l}-1.224^{\star \star *} \\
(0.0402)\end{array}$ & $\begin{array}{c}-0.0456^{\star * *} \\
(0.0245)\end{array}$ & $\begin{array}{l}-1.226^{\star \star *} \\
(0.0402)\end{array}$ \\
\hline Permanente & $\begin{array}{c}0.165 \\
(0.161)\end{array}$ & $\begin{array}{c}-1.406^{\star \star \star} \\
(0.291)\end{array}$ & $\begin{array}{c}0.160 \\
(0.161)\end{array}$ & $\begin{array}{c}-1.373^{\star \star *} \\
(0.292)\end{array}$ \\
\hline Colaborador & $\begin{array}{l}-0.0615 \\
(0.164)\end{array}$ & $\begin{array}{c}-0.712^{\star} \\
(0.294)\end{array}$ & $\begin{array}{l}-0.0676 \\
(0.164)\end{array}$ & $\begin{array}{l}-0.677^{\star} \\
(0.295)\end{array}$ \\
\hline Conceito da Capes & $\begin{array}{c}0.0481^{* * *} \\
(0.0120)\end{array}$ & $\begin{array}{l}-0.122^{\star \star *} \\
(0.0179)\end{array}$ & $\begin{array}{c}0.0489^{* * *} \\
(0.0121)\end{array}$ & $\begin{array}{l}-0.126^{* * *} \\
(0.0180)\end{array}$ \\
\hline Data de criação & $\begin{array}{c}-0.0036^{\star * *} \\
(0.0010)\end{array}$ & $\begin{array}{l}0.0042^{\star *} \\
(0.0014)\end{array}$ & $\begin{array}{c}-0.0035^{\star \star *} \\
(0.001)\end{array}$ & $\begin{array}{l}0.0039^{* *} \\
(0.0014)\end{array}$ \\
\hline Capital & $\begin{array}{l}-0.0114 \\
(0.0247)\end{array}$ & $\begin{array}{c}0.0718 \\
(0.0372)\end{array}$ & $\begin{array}{l}-0.0085 \\
(0.0251)\end{array}$ & $\begin{array}{c}0.0589 \\
(0.0375)\end{array}$ \\
\hline Ciências Agrárias & $\begin{array}{l}0.186^{\star \star \star} \\
(0.0515)\end{array}$ & $\begin{array}{c}-0.443^{* \star *} \\
(0.0829)\end{array}$ & $\begin{array}{l}0.186^{\star \star *} \\
(0.0515)\end{array}$ & $\begin{array}{l}-0.441^{* * *} \\
(0.0829)\end{array}$ \\
\hline Ciências Biológicas & $\begin{array}{l}-0.109^{*} \\
(0.0538)\end{array}$ & $\begin{array}{c}0.0677 \\
(0.0849)\end{array}$ & $\begin{array}{c}-0.110^{*} \\
(0.0539)\end{array}$ & $\begin{array}{c}0.0750 \\
(0.0850)\end{array}$ \\
\hline Ciências da Saúde & $\begin{array}{l}-0.148^{* *} \\
(0.0505)\end{array}$ & $\begin{array}{l}-0.0395 \\
(0.0759)\end{array}$ & $\begin{array}{l}-0.149^{* *} \\
(0.0505)\end{array}$ & $\begin{array}{l}-0.0338 \\
(0.0760)\end{array}$ \\
\hline Ciências Exatas e da Terra & $\begin{array}{l}-0.468^{\star \star \star} \\
(0.0566)\end{array}$ & $\begin{array}{l}0.681^{* \star *} \\
(0.0821)\end{array}$ & $\begin{array}{c}-0.468^{\star \star \star} \\
(0.0566)\end{array}$ & $\begin{array}{l}0.680^{\star * \star} \\
(0.0821)\end{array}$ \\
\hline Ciências Humanas & $\begin{array}{l}-0.0276 \\
(0.0517)\end{array}$ & $\begin{array}{c}0.0976 \\
(0.0769)\end{array}$ & $\begin{array}{l}-0.0292 \\
(0.0518)\end{array}$ & $\begin{array}{c}0.106 \\
(0.0770)\end{array}$ \\
\hline Ciências Sociais Aplicadas & $\begin{array}{l}-0.237^{\star \star \star} \\
(0.0620)\end{array}$ & $\begin{array}{c}0.224^{\star} \\
(0.0884)\end{array}$ & $\begin{array}{l}-0.235^{\star \star \star} \\
(0.0620)\end{array}$ & $\begin{array}{c}0.220^{*} \\
(0.0884)\end{array}$ \\
\hline Engenharia e Informática & $\begin{array}{l}-0.0684 \\
(0.0589)\end{array}$ & $\begin{array}{c}0.0396 \\
(0.0878)\end{array}$ & $\begin{array}{l}-0.0662 \\
(0.0590)\end{array}$ & $\begin{array}{c}0.0268 \\
(0.0877)\end{array}$ \\
\hline Linguística, letras e artes & $\begin{array}{c}-0.116 \\
(0.0609)\end{array}$ & $\begin{array}{c}0.257^{\star \star} \\
(0.0857)\end{array}$ & $\begin{array}{c}-0.115 \\
(0.0609)\end{array}$ & $\begin{array}{l}0.258^{* *} \\
(0.0876)\end{array}$ \\
\hline Doutorado internacional & & & $\begin{array}{l}-0.0308 \\
(0.0344)\end{array}$ & $\begin{array}{l}0.164^{\star \star} \\
(0.0553)\end{array}$ \\
\hline Endógenos & $\begin{array}{c}0.0153 \\
(0.0256)\end{array}$ & $\begin{array}{l}0.101^{* * *} \\
(0.0376)\end{array}$ & & \\
\hline Não endógenos & & & $\begin{array}{l}-0.0050 \\
(0.0278)\end{array}$ & $\begin{array}{l}-0.146^{\star \star \star} \\
(0.0399)\end{array}$ \\
\hline Log-Likelihood & & & & \\
\hline AIC & & & & \\
\hline $\mathrm{N}$ & & & & \\
\hline
\end{tabular}

Fonte: Elaborado pelos autores a partir dos dados da pesquisa.

Nota: Desvio-padrão entre parênteses; ${ }^{*} \mathrm{p}<0.05,{ }^{* *} \mathrm{p}<0.01,{ }^{* * *} \mathrm{p}<0.001$. 